\title{
Analyse Microlocale sur les Variêtếs de Cauchy- Riemann et Problème du Prolongement des Solutions Holomorphes des Équations aux Dérivées Partielles
}

\author{
par \\ Shinichi TAJIMA*
}

Table des Matières.

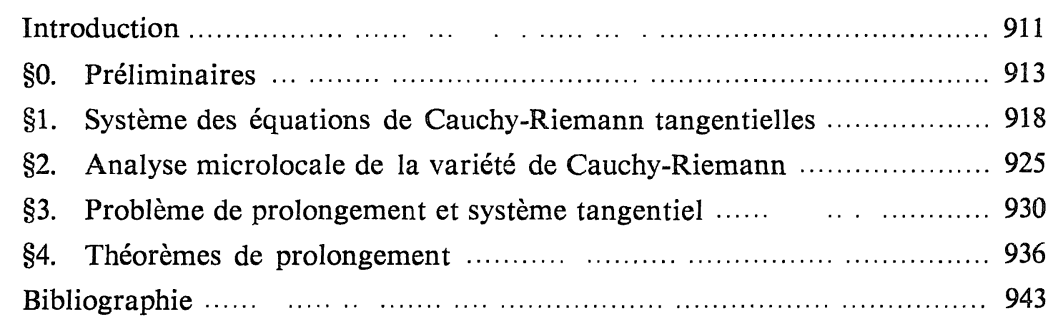

\section{Introduction}

Nous allons nous intéresser au problème suivant: Etant donné une famille finie $\left(P_{j}\right)$ d'opérateurs différentiels à coefficients holomorphes, les solutions holomorphes du système $P_{j} u=0$ définies dans un ouvert $\Omega$ se prolongent-elles en solutions holomorphes au voisinage d'un point frontière de $\Omega$ ?

M. Zerner [45] (dans le cas d'un seul opérateur) et J. M. Bony-P. Schapira [6] ont montré que si le bord de l'ouvert est non caractéristique par rapport à $\left(P_{j}\right)$, toutes les solutions holomorphes se prolongent. Récemment ce résultat a été généralisé par M. Kashiwara [17], M. Kashiwara-T. Kawai [21] et par M. Kashiwara-P. Schapira [22].

C. O. Kiselman [26] a résolu ce problème dans le cas d'un seul opérateur à coefficients constants. P. Pallu de la Barrière [34] et Y. Tsuno [40]-[43] ont

Communiqué par S. Matsuura, le 25 Mars, 1981.

* Ancienne adresse: Département de Mathématiques, Université de Tokyo, Bunkyo-ku, Tokyo, 113 Japon. Nouvelle adresse: Département de Mathématiques, Université de Niigata, Ninomachi 8050, Ikarashi, Niigata, 950-21, Japon. 
très sérieusement étudié le cas d'un seul opérateur $P$ à coefficients holomorphes.

En utilisant la théorie des hyperfonctions et des microfonctions, P. Pallu de la Barrière a montré que si $P$ n'est pas dégénéré au bord, la structure d'obstructions du prolongement est isomorphe à la structure du faisceau des solutions microfonctions d'un système tangentiel.

J. Persson [35] et Y. Tsuno [42], [43] ont abordé l'étude du cas où le bord n'est pas simplement caractéristique pour $P$.

Dans cet article, on interprète le problème du prolongement des solutions holomorphes du point de vue microlocal, en généralisant ce problème pour l'étude systématique.

En ce qui concerne la théorie des systèmes d'équations aux dérivées partielles, on utilisera la théorie des hyperfonctions et des microfonctions de Sato-KawaiKashiwara [37]. En ce qui concerne la théorie des variétés ce Cauchy-Riemann, on aura besoin des résultats de I. Naruki [32], [33] et de A. Andreotti-G. A. Fredricks [1]. On en donnera brièvement un résumé dans le paragraphe 0 .

Le paragraphe 1 est consacré à l'étude du système des équations de CauchyRiemann tangentielles. En particulier nous montrerons que le problème du prolongement (du type "edge of the wedge theorem") des fonctions holomorphes pour la sous variété analytique réelle, générique est équivalent au problème de l'hypo-ellipticité analytique du système de Cauchy-Riemann tangentiel (Th. 1.2.2).

Le paragraphe 2 est consacré à l'étude de la structure microlocale de la sous-variété générique dans la variété analytique complexe. En utilisant les résultats de $\mathrm{S}-\mathrm{K}-\mathrm{K}$, nous étudierons la structure des solutions microfonctions $\mathrm{du}$ système des équations de Cauchy-Riemann tangentielles. En particulier nous retrouverons un résultat de I. Naruki [33] du point de vue microlocal (Th. 2.2.2).

Dans le paragraphe 3, nous traiterons le problème du prolongement (du type "edge of the wedge theorem") des solutions holomorphes du système d'équations aux dérivées partielles. Nous montrerons que la structure d'obstructions du prolongement (pour la sous-variété analytique réelle, générique, de codimension quelconque) est quasi-isomorphe a la structure du faisceau des solutions microfonctions du système tangentiel (Th. 3.1.4). En traitant le problème du prolongement dans le domaine complexe, nous obtiendrons ce résultat de façon naturelle. 
Remarquons ici que notre l'étude a été inspirée par l'article [34] de P. Pallu de la Barrière et aussi par les articles [18] [19] de M. Kashiwara-T. Kawai. En effet, M. Kashiwara-T. Kawai ont traité le problème du prolongement des solutions du système elliptique dans le domaine réel.

Dans le paragraphe 4, on donnera comme applications du théorème 3.1.4 des nouveaux résultats, en mettant à exécution les principes et les procédés dûs à M. Kashiwara-T. Kawai [20] dans certaines cas concrèts. On retrouvra le résultat de J. M. Bony-P. Schapira [6]. On pourra aussi généraliser certains résultats de Y. Tsuno [40], [41] et de P. Pallu de la Barrière [34] au système d'équations.

On étudiera la non-hypo-ellipticité analytique et aussi donnera une methode pour déterminer la structure du faisceau des solutions microfonctions du système tangentiel (Th. 4.2.4 et Th. 4.2.6).

On utilisera le langage des cathégories dérivées. On renvoie à [9] pour les définitions.

\section{§0. Prélimimaires.}

A) Soit $\mathbb{X}$ une variété analytique complexe, munie du faisceau $\mathcal{O}_{\mathbb{X}}$ des fonctions holomorphes. On note $\mathbb{T}^{*} \mathbb{X}$ le fibré vectoriel cotangent à $\mathbb{X}, \mathbb{P}^{*} \mathbb{X}$ le fibré projectif complexe associé.

Le faisceau d'anneau $\mathscr{D}_{\mathbf{X}}$ des opérateurs différentiels sur $\mathbb{X}$ est défini par:

$$
\mathscr{D}_{\mathbf{X}}=\mathscr{H}_{\mathbf{X}}^{\operatorname{dim} \mathbf{X}}\left(\mathcal{O}_{\mathbf{X} \times \mathbf{X}}^{(0, \operatorname{dim} X)}\right)^{f},
$$

où on identifie $\mathbb{X}$ à la diagonale de $\mathbb{X} \times \mathbb{X}$.

On note $v$ la projection $\mathbb{T}^{*} \mathbb{X}-\mathbb{T}_{\mathbf{X}}^{*} \mathbb{X} \rightarrow \mathbb{P} * \mathbb{X}, \pi_{\boldsymbol{X} \mid \mathbf{X} \times \mathbf{X}}$ la projection

$$
(\mathbb{X} \times \mathbb{X}-\mathbb{X})\left\llcorner\mathbb{T}_{\mathbb{X}}^{*}(\mathbb{X} \times \mathbb{X}) \rightarrow \mathbb{X}\right.
$$

le premier espace étant muni de la topologie de co-éclaté ([37], [17]).

On définit le faisceau $\mathscr{E}_{\boldsymbol{X}}$ des opérateurs microdifférentiels sur $\mathbb{T} * \mathbb{X}$ par:

$$
\begin{aligned}
& \mathscr{E}_{\mathbf{X}}=v^{-1} v_{*}\left(\mathscr{H}_{\boldsymbol{T}_{\boldsymbol{X}}^{*}(\boldsymbol{X} \times \boldsymbol{X})}^{\operatorname{dim} \underset{X}{X}}\left(\pi_{\boldsymbol{X} \mid \boldsymbol{X} \times \mathbf{X}}^{-1}\left(\mathcal{O}_{\mathbf{X} \times \mathbf{X}}^{(0, \operatorname{dim} \boldsymbol{X})}\right)\right)\right)^{f} \quad \text { sur } \mathbb{T}^{*} \mathbb{X}-\mathbb{T}_{\mathbf{X}}^{*} \mathbb{X}, \\
& \mathscr{E}_{\mathbf{X}}=\mathscr{D}_{\mathbf{X}} \quad \text { sur } \mathbb{T}_{\mathbf{X}}^{*} \mathbb{X} .
\end{aligned}
$$

Remarquons qu'ils sont des faisceaux d'anneaux cohérents. Pour les définitions précises nous renvoyons à [17], [37].

Définition 0.1.1. Soit $\mathscr{M}$ un $\mathscr{D}_{\mathrm{X}}$-module cohérent à gauche. La variété caracteristique de $\mathscr{M}$, notée $S S(\mathscr{M})$, est l'ensemble 


$$
\operatorname{Supp}\left(\mathscr{E}_{\mathbf{X}} \otimes_{\pi^{-1} \mathscr{\mathscr { X }}} \pi^{-1} \mathscr{M}\right) \subseteq \mathbf{T}^{*} \mathbf{X},
$$

où $\pi$ désigne la projection $\mathbb{T} * \mathbb{X} \rightarrow \mathbb{X}$.

On désignera encore par $\mathscr{M}$ le module $\mathscr{E}_{\mathbf{X}} \otimes_{\pi^{-1} \mathscr{D}_{\mathbf{X}}} \pi^{-1} \mathscr{M}$.

Soit $\phi$ une application holomorphe d'une variété $\mathbb{Y}$ dans la variété $\mathbb{X}$. On pose:

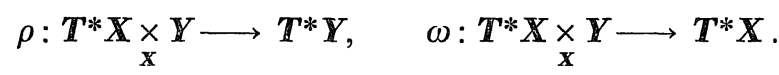

Définition 0.1.2. Soit $\mathscr{M}$ un $\mathscr{D}_{\mathbf{X}}$-module cohérent. On dit que l'application $\phi$ est non caractéristique par rapport à $\mathscr{M}$ si l'application $\rho$ est propre sur $\omega^{-1}(S S(\mathscr{M}))$.

Remarquons que si $\mathbb{Z}$ est une sous-variété analytique complexe de $\mathbb{X}$, cette définition est équivalents à la condition usuelle:

$$
S S(\mathscr{M}) \cap \mathbf{T}_{\mathbf{Y}}^{*} \mathbf{X} \subseteq \mathbb{T}_{\mathbf{X}}^{*} \mathbf{X} .
$$

Le faisceau $\mathscr{D}_{\mathbf{Y} \rightarrow \mathbf{X}}\left(\right.$ resp. $\mathscr{E}_{\mathbf{Y} \rightarrow \mathbf{X}}$ ) est construit dans [15] (resp. dans [37]). $\mathscr{E}_{\boldsymbol{Y} \rightarrow \boldsymbol{X}}$ est un $\rho^{-1} \mathscr{E}_{\boldsymbol{Y}}$-module à gauche et un $\omega^{-1} \mathscr{E}_{\boldsymbol{X}}$-module à droite. On définit un $\mathscr{E}_{\mathbf{Y}}$-module, noté $\phi^{*}(\mathscr{M})$ ou bien $\mathscr{D}_{\mathbf{Y} \rightarrow \mathbf{X}} \otimes_{\mathscr{D}_{\mathbf{X}}} \mathscr{M}$, par:

$$
\rho_{*}\left(\mathscr{E}_{\mathbf{Y} \rightarrow \mathbf{X}} \otimes_{\omega^{-1} \mathscr{E}_{\mathbf{X}}} \omega^{-1} \mathscr{M}\right) .
$$

Théorème 0.1.3 (M. Kashiwara [15], [17]). Soit $\mathscr{M}$ un $\mathscr{D}_{\mathbf{X}}$-module cohérent. Supposons que $\phi$ soit non caractéristique par rapport à $\mathscr{M}$. Alors

(1) $\phi^{*}(\mathscr{K})$ est un $\mathscr{D}_{\mathbf{Y}}$-module cohérent,

(2) $S S\left(\phi^{*}(\mathscr{M})\right)=\rho\left(\omega^{-1}(S S(\mathscr{M}))\right.$.

Dans le cas où $\mathbb{Y}$ est une sous-variété de $\mathbb{X}$, on désignera par $\mathscr{M}_{\mathbf{Y}}$ le module $\phi^{*}(\mathscr{M})$.

Soit $\mathscr{M}$ un $\mathscr{D}_{\mathbf{X}}$-module cohérent à gauche, $\mathscr{F}$ un $\mathscr{D}_{\mathbf{X}}$-module à gauche. Le faisceau $\mathscr{H}_{\text {am } \mathscr{D} X}(\mathscr{M}, \mathscr{F})$ est le faisceau des solutions du système $\mathscr{M}$ à valeurs dans le faisceau $\mathscr{F}$. On note $\mathbb{R} \mathscr{H}_{a m_{\mathscr{D}}}$ le foncteur dérivé du foncteur $\mathscr{H}_{a m_{\mathscr{D}_{X}}}$.

Théorème 0.1.4 (Cauchy-Kowalewski, M. Kashiwara [15], [17]). Soit $\mathscr{M}$ un $\mathscr{D}_{\mathbf{X}}$-module cohérent. Soit $\phi$ une application holomorphe de $\mathbf{Y}$ dans $\mathbf{X}$. Si $\phi$ est non caractéristique par rapport à $\mathscr{M}$, on a alors un isomorphisme naturel:

$$
\phi^{-1}\left(\mathbb{R} \mathscr{H}_{a m_{\mathscr{O}_{X}}}\left(\mathscr{M}, \mathcal{O}_{\mathbf{X}}\right)\right)=\mathbb{R} \mathscr{H}_{a m_{\mathscr{D}_{\mathbf{Y}}}}\left(\mathscr{M}_{\mathbf{Y}}, \mathcal{O}_{\mathbf{Y}}\right)
$$

Soit $\boldsymbol{M}$ une variété analytique réelle de complexifiée $\boldsymbol{X}$. Le faisceau $\mathscr{B}_{M}$ 
des hyperfonctions de Sato est défini par:

$$
\mathscr{B}_{M}=\mathscr{H}_{M}^{\operatorname{dim} M}\left(\mathcal{O}_{X}\right) \otimes \omega_{M \mid X}=\mathbb{R} \Gamma_{M}\left(\mathcal{O}_{\mathbf{X}}\right) \otimes \omega_{M \mid X}[\operatorname{dim} \mathbb{M}],
$$

où $\mathbb{R} \Gamma_{M}$ désigne le foncteur dérivé du foncteur $\Gamma_{M}$ et $\omega_{M \mid X}$ désigne le faisceau de l'orientation relative de $\mathbb{R}$ à $X$.

Théorème 0.1.5 (H. Komatsu-T. Kawai [29], M. Kashiwara [15]). Soit $\mathbb{N}$ une sous-variété analytique réelle de $\mathbb{R}, \mathbb{Z}$ un complexifié de $\mathbb{N}$ dans $\mathbb{X}$. Soit $\mathscr{M}$ un $\mathscr{D}_{\mathbf{X}}$-module cohérent. Si $\mathbb{Y}$ est non caractéristique par rapport à $\mathscr{M}$, on a alors:

$$
\mathbb{R} \Gamma_{N} \mathbb{R} \mathscr{H}_{a m_{\mathscr{D}_{\mathbf{X}}}}\left(\mathscr{M}, \mathscr{B}_{M}\right) \otimes \omega_{N \mid M}=\mathbb{R} \mathscr{H}_{a m_{\mathscr{D}}}\left(\mathscr{M}_{\mathbf{Y}}, \mathscr{B}_{N}\right)\left[-\operatorname{codim}_{M} N\right] .
$$

Soit $\mathbb{R}$ une variété analytique réelle de complexifiée $\mathbb{X}$. Le faisceau $\mathscr{C}_{M}$ des microfonctions sur $S_{M}^{*} \mathbb{X}$ est défini grâce à la transformation comonoïdale par:

$$
\mathscr{C}_{M}=\mathscr{H}_{S_{M}^{*} \mathbf{X}} \operatorname{dim}_{M}\left(\pi^{-1} \mathcal{O}_{\mathbf{X}}\right)^{a} \otimes \omega_{M \mid X}=\mathbb{R} \Gamma_{S_{M}^{+} \mathbf{X}}\left(\pi^{-1} \mathcal{O}_{\mathbf{X}}\right)^{a} \otimes \omega_{M \mid X}[\operatorname{dim} \mathbb{M}],
$$

où $a$ désigne l'application antipodale et $\pi$ la projection

$$
(\mathbb{X}-\mathbb{M}) \sqcup S_{M}^{*} X \longrightarrow \mathbb{X} \text {. }
$$

Remarquons que le faisceau $\mathscr{C}_{M}$ a une structure de $\mathscr{E}_{\mathbf{X}}$-module à gauche.

Définition 0.1.6. Soit $\mathscr{M}$ un $\mathscr{D}_{\mathbf{x}}$-module cohérent. On dit que $\mathscr{M}$ est un système elliptique sur $\mathbb{M}$ si:

$$
S S(\mathscr{M}) \cap \mathbb{T}_{M}^{*} \cong \mathbb{T}_{M}^{*} \mathbb{M}
$$

Théorème 0.1 .7 (S-K-K [37]). Soit une variété analytique réelle de complexifiée $\mathbb{X}$. Soit $\mathscr{M}$ un $\mathscr{D}_{\mathbf{X}}$-module cohérent. Si $\mathscr{M}$ est elliptique sur $\mathbb{R}$, on a alors:

$$
\begin{aligned}
& \mathbb{R} \mathscr{H}_{\text {am }_{\mathbf{X}}}\left(\mathscr{M}, \mathscr{C}_{M}\right)=0, \\
& \mathbb{R} \mathscr{H}_{\text {am }_{\mathscr{D}_{X}}}\left(\mathscr{M}, \mathscr{B}_{M}\right)=\mathbb{R} \mathscr{H}_{a m_{\mathscr{D}_{X}}}\left(\mathscr{M}, \mathscr{A}_{M}\right),
\end{aligned}
$$

où $\mathscr{A}_{M}$ désigne le faisceau des fonctions analytiques réelles sur MI.

B) Dans le paragraphe prochain, on verra que la théorie de la variété de Cauchy-Riemann sera importante pour résoudre le problème du prolongement. En particulier nous utiliserons le résultat de I. Naruki [32], celui de G. Tomassini [39] et de A. Andreotti-G.I. Fredricks [1].

Soit $\mathbb{N}$ une variété analytique réelle, $\mathbb{T N} \otimes \mathbb{C}$ son fibré vectoriel tangent complexifié. 
La structure de Cauchy-Riemann de type $l$ sur $\mathbb{N}$ est un sousespace fibré analytique $\boldsymbol{A}$ de $\mathbb{T} \mathbb{N} \otimes \boldsymbol{C}$ tel que

(0) $\operatorname{dim}_{\boldsymbol{C}} \mathbb{A}_{\boldsymbol{p}}=l \quad p \in \mathbb{N}$

(1) $\bar{A} \cap A=T_{N} \mathbb{N} \otimes \mathbb{C}$,

(2) $\mathbb{A}_{P}$ est involutif $\rho \in \mathbb{N}$.

On dit que $(\mathbb{N}, \boldsymbol{A})$ est la variété de Cauchy-Riemann munie de la structure $\mathbb{A}$ (S. J. Greenfield [8]).

Exemple 0.2.1. Soit $\mathbb{N}$ une variété analytique réelle. Alors $\left(\mathbb{N}, \mathbb{T}_{N} \mathbb{N}\right)$ est une variété de Cauchy-Riemann de type 0 . On l'appelle structure totalement réelle.

Exemple 0.2.2. Soit $\mathbb{X}$ une variété analytique complexe. On désigne par $\mathcal{A} \mathbf{H}(\boldsymbol{X})=\mathbf{T}^{0,1}(\mathbb{X})$ l'espace fibré vectoriel tangent antiholomorphe. Il est clair que $\left(\mathbb{X},(\boldsymbol{A H}(\mathbb{X}))\right.$ est une variété de Cauchy-Riemann de type $\operatorname{dim}_{\boldsymbol{C}} \mathbb{X}$.

Exemple 0.2.3. Soient $\mathbb{N}$ une variété analytique réelle et $\mathbb{X}$ une variété analytique complexe. Soit $\phi$ un plongement analytique de $\mathbb{N}$ dans $\mathbb{X}$. L'application $\phi$ induit un morphisme $\phi_{*}$ de façon naturelle:

$$
\phi_{*}: \mathbb{T N} \otimes \mathbb{C} \longrightarrow \mathbf{T X} \otimes \mathbb{C} .
$$

On pose

$$
A \mathbb{T}_{\phi}(\mathbb{N})=\phi_{*}^{-1}(\mathcal{A H}(\mathbb{X})), h(\rho)=\operatorname{dim}_{C}\left(\phi_{*}^{-1}(\mathcal{A H}(\mathbb{X}))\right)_{p} \quad \text { pour } \quad p \in \mathbb{N} .
$$

Si $h(p)$ est égal à une constante $h$ pour tout $p \in \mathbb{N}$, alors $\left(\mathbb{N}, A \mathbb{T}_{\phi}(\mathbb{N})\right)$ est une variété de Cauchy-Riemann de type $h$.

La proposition suivante est bien connue.

Proposition 0.2.4 (cf. [1]). Soient $\mathbf{X}$ une variété analytique complexe et $\mathbb{N}$ une sous-variété analytique réelle de $\mathbb{X}$. Supposons que $\mathbb{N}$ soit munie de la structure de Cauchy-Riemann de type $h$ induite par $\mathbb{A}(\mathbb{H})$ comme dans l'exemple 0.2.3. On a alors

$$
\operatorname{dim}_{R} \mathbf{N}-\operatorname{dim}_{\boldsymbol{C}} \mathbf{X} \leqq h \leqq \frac{1}{2} \operatorname{dim}_{R} \mathbf{N}
$$

Définition 0.2.5 (S. J. Greenfield [8]). Dans la situation précédente, on dit que $\mathbb{N}$ est générique si on a $h=\operatorname{dim}_{R} \mathbb{N}-\operatorname{dim}_{\boldsymbol{C}} \mathbb{X}$.

Soit $\boldsymbol{N}$ une sous-variété analytique réelle de $\boldsymbol{X}$ définie localement par les équations à valeurs réelles: 


$$
\begin{aligned}
& f_{1}=f_{2}=\cdots \cdots=f_{2 m-n}=0, \\
& d f_{1} \wedge d f_{2} \wedge \cdots \cdots \wedge d f_{2 m-n} \neq 0 \quad \text { sur } \mathbb{N},
\end{aligned}
$$

oú $m=\operatorname{dim}_{C} \mathbb{X}$ et $n=\operatorname{dim}_{R} \mathbb{N}$. Soit $\left(z_{1}, z_{2}, \ldots, z_{m}\right)$ un système de coordonnées holomorphes locales autour de $p \in \mathbb{N}$.

Proposition 0.2.6 (cf. [1]). Les assertions suivantes sont équivalentes:

(1) $\mathbb{N}$ est générique au point $p \in \mathbb{N}$.

(2) $\operatorname{rank}\left\{\frac{\partial\left(f_{1}, f_{2}, \ldots, f_{2 m-n}\right)}{\partial\left(z_{1}, z_{2}, \ldots, z_{m}\right)}\right\}_{p}=2 m-n$.

(3) $\operatorname{rank}\left\{\frac{\partial\left(f_{1}, f_{2}, \ldots, f_{2 m-n}\right)}{\partial\left(\bar{z}_{1}, \bar{z}_{2}, \ldots, \bar{z}_{m}\right)}\right\}_{p}=2 m-n$.

Remarquons que l'hypersurface analytique réelle de $\mathbb{X}$ est générique. La sous-variété analytique complexe n'est pas générique.

Dépunition $\mathbb{0} .2 . \%$. Soit $(\mathbb{N}, \mathbb{A})$ une variété de Cauchy-Riemann. On dit que $(\mathbb{X}, \phi)$ est un complexifié de $(\mathbb{N}, \mathbb{A})$ si:

(1) $\mathbb{X}$ est une variété analytique complexe.

(2) $\phi: \mathbb{N} \rightarrow \mathbb{X}$ est un plongement analytique férmée.

(3) La sous-variété $\phi(\mathbb{N})$ est générique.

(4) $\phi_{*} A=A T_{\phi}(\mathbb{N})$.

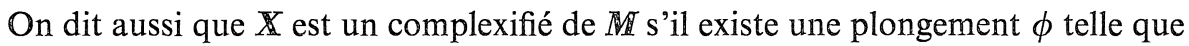
$(\mathbb{X}, \phi)$ est un complexifié de $\left(\mathbb{M}, \mathbb{T}_{M} M\right.$ M $)$.

Théorème 0.2 .8 (I. Naruki [32]. cf. [1]). Soit $(\mathbb{N}, \mathbb{A})$ une variété de Cauchy-Riemann, analytique réelle. Alors

(1) Il existe un complexifié de $(\mathbb{N}, \mathbb{A})$,

(2) Supposons que $(\mathbb{N}, \phi)$ et $(\mathbb{Y}, \psi)$ soient deux complexifiés de $(\mathbb{N}, A)$, alors il existe un voisinage $\mathbb{U}$ de $\phi(\mathbb{N})$ dans $\mathbb{X}$, un voisinage $\mathbb{V}$ de $\psi(\mathbb{N})$ dans $\mathbb{Y}$ et une application biholomorphe

$$
h: \mathbb{U} \longrightarrow \mathbb{V} \text { tels que } h \circ \phi=\psi .
$$

De plus, si $\mathbb{U}$ est suffisamment petit et connexe à $\mathbb{N}$, alors $h$ est uniquement déterminé.

Définition 0.2 .9 (A. Andreotti-G. A. Fredricks [1]). Soit $\mathbb{N}$ une variété analytique réelle. Soient $A, \mathbb{B}$ deux structures de Cauchy-Riemann sur $\mathbb{N}$. On dit que $\mathbb{B}$ domine $A$ si:

$$
A_{p} \subseteq \mathbb{R}_{p} \quad \text { quel que soit } \quad p \in \mathbb{N} .
$$


Théorème $\mathbf{0 . 2 . 1 0}$ (A. Andreotti-G. A. Fredricks [1], cf. G. Tommassini [39]). Dans les conditions précédentes, $(\mathbb{X}, \phi)$ et $(\mathbb{Z}, \psi)$ sont des complexifiés de $(\boldsymbol{N}, \mathcal{A})$ et de $(\boldsymbol{N}, \boldsymbol{B})$ respectivement. On a les résultats suivants:

(1) Il existe un voisinage $\mathbb{U}$ de $\phi(\mathbf{N})$ dans $\mathbb{X}$, un voisinage $\boldsymbol{V}$ de $\psi(\boldsymbol{N})$ et une submersion holomorphe

$$
h: \mathbb{U} \longrightarrow \mathbb{V} \text { tels que } h \circ \phi=\psi .
$$

(2) Si U est suffisamment petit et connexe à $\phi(\mathbb{N})$, alors l'application $h$ se détermine uniquement.

\section{§1. Système des Équations de Cauchy-Riemann Tangentielles}

A) Etant donnée une variété de Cauchy-Riemann $(\mathbb{N}, \boldsymbol{A})$ nous allons interpréter la structure de Cauchy-Riemann comme un système d'équations linéaires aux dérivées partielles.

Nous montrerons grâce à l'existence d'un complexifié $(\boldsymbol{X}, \phi)$ que le système d'équations aux dérivées partielles associé à $\mathbb{A}$ est isomorphe au système tangentiel pour le système des équations de Cauchy-Riemann sur $\boldsymbol{X}$.

Soit $\mathbb{X}$ une variété analytique complexe, munie du faisceau $\mathcal{O}_{\boldsymbol{X}}$ des fonctions holomorphes. Soit $\boldsymbol{X}_{R}$ la variété analytique réelle sous-jacente à $\mathbb{X}, \boldsymbol{X}_{C}$ un complexifié de $\boldsymbol{X}_{R}$.

On désigne par $\mathscr{D}_{C-R}$ le système des équations de Cauchy-Riemann sur $\mathbf{X}_{R}$. C'est un $\mathscr{D}_{\mathbf{X}_{C}}$-module cohérent.

Nous montrons ici un résultat essentiels.

Théorème 1.1.1. Soit $\mathbb{N}$ une sous-variété analytique réelle de $\mathbb{X}$. Soit $\mathbf{Y}$ un complexifié de $\mathbf{N}$ dans $\mathbf{X}$.

Pour que $\boldsymbol{Y}$ soit non caractéristique par rapport à $\mathscr{D}_{C-R}$ au voisinage de $\mathbb{N}$, il faut et il suffit que $\mathbb{N}$ soit générique dans $\mathbf{X}$.

Démonstration. La question étant locale, prenons un point $p \in \mathbb{N}$. Soit $(z)=(x+i y)$ un système de coordonnées holomorphes au voisinage de $p$ dans $\boldsymbol{X}$. Soient $f_{1}(x, y)=f_{2}(x, y)=\cdots=f_{2 m-n}(x, y)=0$ les équations analytique réelles de $\mathbb{N}$ au voisinage de $p$ dans $\boldsymbol{X}$, i.e.

$$
\begin{aligned}
& \mathbf{N}=\left\{(x+i y) \in \boldsymbol{X} \mid f_{1}(x, y)=f_{2}(x, y)=\cdots=f_{2 m-n}(x, y)=0\right\} \\
& \text { où } \quad d f_{1}(x, y) \wedge d f_{2}(x, y) \wedge \cdots \wedge d f_{2 m-n}(x, y) \neq 0 \quad \text { sur } \quad \mathbb{N} .
\end{aligned}
$$


Soit $(\tilde{x}, \tilde{y})=(x+i \hat{x}, y+i \hat{y})$ le complexifié de $(x, y)$. Désignons par $\tilde{f}_{j}(\tilde{x}, \tilde{y})$ les complexifiés de $f_{j}(x, y)$ dans $\mathbb{X}_{C}$. On obtient que

$$
\mathbb{Y}=\left\{(\tilde{x}, \tilde{y}) \in \mathbb{X}_{C} \mid \tilde{f}_{1}(\tilde{x}, \tilde{y})=\tilde{f}_{2}(\tilde{x}, \tilde{y})=\cdots=\tilde{f}_{2 m-n}(\tilde{x}, \tilde{y})=0\right\}
$$

au voisinage de $p$ dans $\mathbb{X}_{C}$.

Rappelons la définition 0.1.2: $\mathbb{Y}$ est non caractéristique par rapport à $\mathscr{D}_{C-R}$ si $S S\left(\mathscr{D}_{C-R}\right) \cap \mathbb{T}_{\boldsymbol{Y}}^{*}\left(\mathbb{X}_{C}\right) \subseteq \mathbb{T}_{\boldsymbol{X}_{C}}^{*}\left(\mathbb{X}_{C}\right)$.

Soit $(\tilde{x}, \tilde{y} ; \tilde{\xi}, \tilde{\eta})$ le système de coordonnées symplectiques de $\mathbb{T}^{*}\left(\mathbb{X}_{C}\right)$ au voisinage de $p$. Il est facile de voir que $(\tilde{x}, \tilde{y} ; \tilde{\xi}, \tilde{\eta})$ appartient à $S S\left(\mathscr{D}_{C-R}\right)$ $\cap \mathbb{T}_{\mathbf{Y}}^{*}\left(\mathbb{X}_{C}\right)$ si et seulement si :

i) $f_{j}(x, y)=0 \quad j=1,2, \ldots, 2 m-n$,

ii) $\alpha_{1}, \alpha_{2}, \ldots, \alpha_{2 m-n} \in C$ tels que

$\tilde{\xi}_{j}+i \tilde{\eta}_{j}=\tilde{\partial}_{\bar{z}_{j}}\left\{\alpha_{1} \tilde{f}_{1}(\tilde{x}, \tilde{y})+\cdots+\alpha_{2 m-n} \tilde{f}_{2 m-n}(\tilde{x}, \tilde{y})\right\}=0 \quad j=1,2, \ldots, 2 m-n$, ò̀ $\quad \tilde{\partial}_{\bar{z}_{j}}=\frac{1}{2}\left(\frac{\partial}{\partial \tilde{x}}+i \frac{\partial}{\partial \tilde{y}}\right)$.

On en dèduit que $Y$ est non caractéristique par rapport à $\mathscr{D}_{C-R}$ si et seulement si:

$$
\operatorname{rank}\left\{\frac{\tilde{\partial}\left(f_{1}, f_{2}, \ldots, f_{2 m-n}\right)}{\tilde{\partial}\left(z_{1}, z_{2}, \ldots, z_{m}\right)}\right\}=2 m-n \quad \text { sur } \mathbb{Y} .
$$

D'autre part on a $\tilde{\partial}_{\bar{z}_{J}} \tilde{f}_{k}=\partial_{\bar{z}_{J}} f_{k}(j=1,2, \ldots, m, k=1,2, \ldots, 2 m-n)$ sur $\mathbb{X}$.

Il est clair d'après ce qui précède que la proposition 0.2 .6 entraîne le théorème 1.1.1.

c. q.f.d.

Soit $\mathbb{N}$ une variété analytique réelle de complexifiée $\mathbb{Y}$. Supposons que $\mathbb{N}$ se munisse d'une structure de Cauchy-Riemann $\mathscr{A}$. On désigne par $\mathscr{Q}$ l'idéal de l'anneau $\mathscr{D}_{\boldsymbol{Y}} \mid \mathbb{N}$ engendré par $\Gamma^{\omega}(\mathbb{A})$. Le complexifié de $\left(\mathscr{D}_{\boldsymbol{Y}} \mid \mathbb{N}\right) / \mathscr{Q}$, noté $\mathscr{S}_{C-R, A}$, est un $\mathscr{D}_{\mathbf{Y}}$-module cohérent.

Exemple 1.1.2. Munissons une variété analytique réelle $\mathbb{R}$ de la structure totalement réelle. Soit un complexifié de $\mathbb{M}$. On a alors $\mathscr{S}_{C-R, T_{M M}}=\mathscr{D}_{X}$.

Exemple 1.1.3. Soit $\mathbb{X}$ une variété analytique complexe. (X, $\mathbb{A}(\mathbb{H}))$ est une variété de Cauchy-Riemann. On a alors

$$
\mathscr{S}_{C-R, A H(X)}=\mathscr{D}_{C-R} .
$$

Le résultat suivant est une conséquence du théorème 1.1.1 et du résultat de I. Naruki (le théorème 0.2 .8 ). Grâce à ce résultat, on peut étudier la variété de Cauchy-Riemann du point de vue de S-K-K. 
Théorème 1.1.4. Soit $\mathbf{N}$ une variété analytique réelle de complexifiée $\mathbb{Y}$. Soit $\mathbb{A}$ une structure de Cauchy-Riemann sur $\mathbb{N},(\mathbb{X}, \phi)$ un complexifié de $(\mathbb{N}, \mathcal{A})$. On note $\phi_{C}$ le complexifié de $\phi$, i.e. $\phi_{C}: \mathbb{Y} \rightarrow \mathbb{X}_{C}$.

Alors le système $\mathscr{S}_{C-R, A}$ est isomorphe à un système des équations de Cauchy-Riemann tangentielles. Plus précisément on a

$$
\mathscr{S}_{C-R, A}=\phi_{C}^{*}\left(\mathscr{D}_{C-R}\right)=\mathscr{D}_{\mathbf{Y} \rightarrow \mathbf{X}_{C}} \otimes_{\mathscr{D}_{\mathbf{X}_{C}}} \mathscr{D}_{C-R} \quad \text { en tant que } \mathscr{D}_{\mathbf{Y}} \text {-modules. }
$$

Démonstration. Par abus de langage nous désignerons encore par $\phi$ le complexifié de $\phi$, i.e. $\phi: \mathbb{Z} \rightarrow \mathbb{X}$. L'application $\phi$ étant un plongement on a alors:

$$
\mathscr{S}_{C-R, A}=\phi^{*}\left(\mathscr{S}_{C-R, \phi_{*}(A)}\right) .
$$

La structure $\phi_{*}(\boldsymbol{A})$ coïncide avec la structure induie par $\boldsymbol{A H}(\boldsymbol{X})$ ce qui entraîne que

$$
\mathscr{S}_{C-R, \phi_{*(\boldsymbol{A})}}=\mathscr{D}_{C-R \mid \phi(\boldsymbol{Y})} \quad \text { (cf. le lemme 2.1.5). }
$$

On obtient donc

$$
\mathscr{S}_{C-R, A}=\phi^{*}\left(\mathscr{D}_{C-R \mid \phi(\mathbf{Y})}\right)=\phi_{C}^{*}\left(\mathscr{D}_{C-R}\right) . \quad \text { c. q.f.d. }
$$

Soit $\boldsymbol{X}$ une variété analytique complexe, $\overline{\mathbb{X}}$ le conjugué complexe de $\boldsymbol{X}$. Si on identifie $\boldsymbol{X}_{R}$ avec la diagonale de $\boldsymbol{X} \times \overline{\mathbf{X}}, \boldsymbol{X} \times \overline{\mathbf{X}}$ est un complexifié de $\boldsymbol{X}_{R}$. On désigne par $\tau$ la projection de $\mathbb{X} \times \overline{\mathbb{X}}$ sur

Par définition le système des équations de Cauchy-Riemann sur $\mathbb{X}_{R}$ coïncide avec le $\mathscr{D}_{\mathbf{X} \times \overline{\mathbf{X}}}$-module suivant:

$$
\tau * \mathscr{D}_{\mathbf{X}}=\mathscr{D}_{\mathbf{X} \times \overline{\mathbf{X}} \rightarrow \mathbf{X}} \otimes_{\mathscr{D}_{\mathbf{X}}} \mathscr{D}_{\mathbf{X}}
$$

Lemme 1.1.5. Soit $\mathbb{N}$ une variété analytique réelle, générique de $\mathbb{X}$. Soit $\mathbb{Y}$ un complexifié de $\mathbb{N}$ dans $\mathbb{X} \times \overline{\mathbb{X}}$. On désigne par $\phi$ l'inclusion $\phi$ : $\mathbb{N} \rightarrow \mathbb{X}$, et par $\phi_{C}: \mathbf{Y} \rightarrow \mathbb{X} \times \overline{\mathbf{X}}$ le complexifié de $\phi$. On a alors

(1) $\tau \circ \phi_{C} \mid \mathbb{N}: \mathbf{N} \rightarrow \mathbb{X}$ coincide avec $\phi: \mathbb{N} \rightarrow \mathbf{X}$.

(2) $\tau \circ \phi_{C}: \mathbb{Y} \rightarrow \mathbb{X}$ est une submersion.

Démonstration. Choisissons un système de coordonnées locales $(s+i t)$ holomorphes sur $\mathbb{Y}$, réelles sur $\boldsymbol{N}$. Il est facile de voir que

$$
\phi_{C}(s+i t)=(\phi(s+i t), \bar{\phi}(s+i t)) \text {. }
$$

Cela achève la démonstration de (1). On déduit de (1) et du théorème 0.2 .10 le résultat (2) immédiatement. 
Par abus de langage nous désignerons encore par $\phi$ le composé $\tau_{\circ} \phi_{C}: \mathbb{Y} \rightarrow \mathbb{X}$.

Lemme 1.1.6. Soit $\mathbb{N}$ une sous-variété analytique réelle, générique de $\mathbb{X}$. Soit $\mathbb{Y}$ un complexifié de $\mathbb{N}$ dans $\mathbb{X} \times \overline{\mathbf{X}}$. On désigne par $\mathscr{D}_{C-R}$ le système des équations de Cauchy-Riemann. On a alors

$$
\mathscr{D}_{C-R \mid \mathbf{Y}}=\phi^{*}\left(\mathscr{D}_{\mathbf{X}}\right)=\mathscr{D}_{\mathbf{Y} \rightarrow \mathbf{X}} \otimes_{\mathscr{D}_{\mathbf{X}}} \mathscr{D}_{\mathbf{X}}
$$

Démonstration. Par définition on a

$$
\begin{aligned}
\mathscr{D}_{C-R \mid \mathbf{Y}} & =\phi_{C}^{*}\left(\mathscr{D}_{C-R}\right)=\phi_{C}^{*}\left(\tau^{*}\left(\mathscr{D}_{\mathbf{X}}\right)\right) \\
& =\mathscr{D}_{\mathbf{Y} \rightarrow \mathbf{X} \times \overline{\mathbf{X}}} \otimes_{\mathscr{D}_{\mathbf{X}} \times \overline{\mathbf{X}}} \mathscr{D}_{\mathbf{X} \times \overline{\mathbf{X}} \rightarrow \mathbf{X}} \otimes_{\mathscr{D}_{\mathbf{X}}} \mathscr{D}_{\mathbf{X}} .
\end{aligned}
$$

Un résultat de S-K-K (Chap. 2, Lemme 3.5.1) entraine que

$$
\phi_{C}^{*}\left(\tau^{*}\left(\mathscr{D}_{\mathbf{X}}\right)\right)=\left(\tau \circ \phi_{C}\right)^{*} \mathscr{D}_{\mathbf{X}}=\phi^{*} \mathscr{D}_{\mathbf{X}} .
$$

L'application $\phi: \mathbb{Y} \rightarrow \mathbb{X}$ étant une submersion, le résultat suivant est une conséquence immédiate du lemme 1.1.6.

Proposition 1.1.7. Soit $\mathbb{N}$ une sous-variété analytique réelle et que générique dans $\mathbb{X}$. Soit $\mathbb{Y}$ un complexifié de $\mathbb{N}$ dans $\mathbb{X} \times \overline{\mathbb{X}}$. On obtient alors

$$
S S\left(\mathscr{D}_{C-R \mid Y}\right)=\phi^{-1}\left(T^{*} \mathbb{X}\right)=(\mathbb{T} * \mathbb{X}) \underset{\mathbf{X}}{\times} \mathbb{Y} .
$$

B) Soit $\mathbb{N}$ une sous-variété analytique réelle, générique de $\mathbb{X}$. Nous traiterons le problème du prolongement des fonctions holomorphes à la sous-variété $\mathbb{N}$.

Nous montrerons que la structure d'obstructions du prolongement est isomorphe au faisceau des solutions microfonctions du système des équations de Cauchy-Riemann tangentielles sur $\mathbb{N}$.

On obtient d'abord la

Proposition 1.2.1. Soit $\mathbb{N}$ une sous-variété analytique réelle, générique de $\mathbb{X}$. Soit $\mathbb{Y}$ un complexifié de $\mathbb{N}$ dans $\mathbb{X} \times \overline{\mathbb{X}}$. On note $\phi$ la submersion de $\mathbb{Y}$ sur $\mathbb{X}$. On a alors

$$
S S\left(\mathscr{D}_{C-R \mid Y}\right) \cap \mathbb{T}_{N}^{*} \mathbb{Y}=\phi^{*}\left(\mathbb{T}_{N}^{*} \mathbb{X}\right) .
$$

Démonstration. On obtient que le diagramme suivant est commutatif.

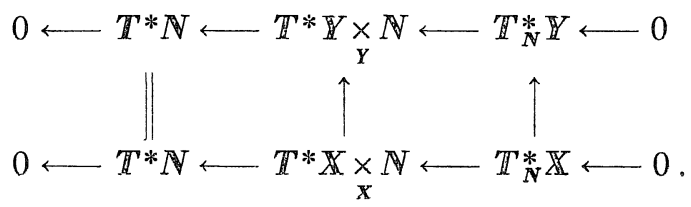


D'après la proposition 1.1 .7 , on a $S S\left(\mathscr{D}_{C-R \mid Y}\right)=\phi^{*}(\mathbb{T} * \mathbf{X})$. On en déduit le résultat immédiatement. c. q.f.d.

Nous allons identifier la variété $T_{N}^{*} X$ avec une sous-variété de $T_{N}^{*} Y$. Nous obtenons enfin le

Théorème 1.2.2. Soient $\mathbb{X}$ une variété analytique complexe et $\mathbb{N}$ une sous-variété analytique réelle, générique de $\boldsymbol{X}$. On désigne par $\mathbb{Y}$ un complexifié de $\mathbf{N}$ dans $\mathbf{X} \times \overline{\mathbf{X}}$. On a alors les quasi-isomorphismes suivantes:

(1) $\mathbf{R} \mathscr{H}_{a m_{\mathscr{D}}}\left(\mathscr{D}_{C-R \mid \mathbf{Y}}, \mathscr{A}_{\mathbf{N}}\right)=\mathcal{O}_{\mathbf{X}} \mid \mathbb{N}$.

(2) $\mathbf{R} \mathscr{H}_{a m_{\mathscr{D}_{\mathbf{Y}}}}\left(\mathscr{D}_{C-R \mid \mathbf{Y}}, \mathscr{B}_{\boldsymbol{N}}\right)\left[-\operatorname{codim}_{\mathbf{X}} N\right]=\mathbf{R} \Gamma_{\boldsymbol{N}}\left(\mathcal{O}_{\mathbf{X}}\right) \otimes \omega_{N \mid \mathbf{X}}$

(3) $\mathbf{R} \mathscr{H}_{a m_{\mathscr{V}}}\left(\mathscr{D}_{C-R \mid \mathbf{Y}}, \mathscr{C}_{N}\right)\left[-\operatorname{codim}_{X} N\right]=\mathbb{R} \Gamma_{S_{N}^{*} X}\left(\pi_{N \mid X}^{-1}\left(\mathcal{O}_{X}\right)\right)^{a} \otimes \omega_{N \mid X}$.

Ici on a posé:

$\mathcal{O}_{\mathbf{X}}$ : le faisceau des fonctions holomorphes sur $\mathbf{X}$.

$\mathscr{A}_{N}$ : le faisceau des fonctions analytiques réelles sur $\mathbb{N}$.

$\mathscr{B}_{\mathbf{N}}$ : le faisceau des hyperfonctions sur $\mathbf{N}$.

$\mathscr{C}_{\mathbf{N}}$ : le faisceau des microfonctions sur $\mathbf{S}_{\boldsymbol{N}}^{*} \mathbf{Y}$.

a: l'application antipodale $\boldsymbol{S}_{\boldsymbol{N}}^{*} \boldsymbol{Y} \rightarrow \boldsymbol{S}_{\boldsymbol{N}}^{*} \boldsymbol{Y}$.

$\pi_{\boldsymbol{N} \mid \mathbf{X}}:$ la projection $(\boldsymbol{X}-\mathbb{N}) \sqcup \mathbf{S}_{\mathbf{N}}^{*} \mathbf{X} \rightarrow \mathbf{X}$.

Remarquons que le théorème 1.2.2 est un prototype du théorème 3.1.4.

Démonstration. Puisque l'application $\phi: \mathbb{Y} \rightarrow \mathbb{X}$ est une submersion, on a l'isomorphisme suivant:

$$
\mathbf{R} \mathscr{H}_{a m_{\mathscr{D}_{\mathbf{Y}}}}\left(\mathscr{D}_{\mathbf{Y} \rightarrow \mathbf{X}} \otimes_{\mathscr{D}_{\mathbf{X}}} \mathscr{D}_{\mathbf{X}}, \mathcal{O}_{\mathbf{X}}\right)=\phi^{-1} \mathbf{R} \mathscr{H}_{a m_{\mathscr{D}_{X}}}\left(\mathscr{D}_{\mathbf{X}}, \mathcal{O}_{\mathbf{X}}\right)
$$

Le lemme 1.1 .6 entraine que

$$
\mathbf{R} \mathscr{H}_{a m_{D_{\mathbf{Y}}}}\left(\mathscr{D}_{C-R \mid \mathbf{Y}}, \mathcal{O}_{\mathbf{Y}}\right)=\phi^{-1} \mathcal{O}_{\mathbf{X}} .
$$

On déduit du lemme 1.1.5 le résultat immédiatement.

(2) D'après le théorème 0.1 .5 , on a

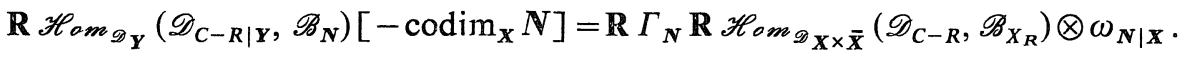

Il est facile de voir que

$$
\mathbf{R} \mathscr{H}_{a m_{\mathscr{D}} \times \overline{\mathbf{X}}}\left(\mathscr{D}_{C-R}, \mathscr{B}_{X_{R}}\right)=\mathcal{O}_{\mathbf{X}}
$$

On en déduit le résultat tout de suite.

(3) (cf. S-K-K. Chap. 3, Th. 2.2.5). 
On désigne par $\pi$ la projection $\pi:(\mathbb{Y}-\mathbb{N}) \sqcup \mathbb{S}_{N}^{*} \mathbb{Y} \rightarrow \mathbb{Y}$. En utilisant un résultal de S-K-K (Chap. 1, Lemme 2.2.3) on obtient que

$$
\begin{aligned}
& \mathbb{R} \Gamma_{S_{N}^{*} \mathbf{X}}\left(\pi_{\boldsymbol{N} \mid \mathbf{X}}^{-1}\left(\mathcal{O}_{\mathbf{X}}\right)\right)^{a} \otimes \omega_{N \mid \mathbf{X}}\left[\operatorname{codim}_{\mathbf{X}} \mathbb{N}\right] \\
& =\mathbb{R} \Gamma_{S_{N}^{*} \mathbf{Y}}\left(\pi^{-1} \phi^{-1}\left(\mathcal{O}_{\mathbf{X}}\right)\right)^{a} \otimes \omega_{N \mid \mathbf{Y}}\left[\operatorname{codim}_{\mathbf{Y}} \mathbb{N}\right] \\
& =\mathbb{R} \Gamma_{S_{N}^{*} \mathbf{Y}}\left(\pi^{-1} \mathbb{R} \mathscr{H}_{a m_{\mathscr{D}_{\mathbf{Y}}}}\left(\mathscr{D}_{\mathbf{C}-\mathbf{R} \mid \mathbf{Y}}, \mathcal{O}_{\mathbf{Y}}\right)\right)^{a} \otimes \omega_{N \mid \mathbf{Y}}\left[\operatorname{codim}_{\mathbf{Y}} \mathbb{N}\right]
\end{aligned}
$$

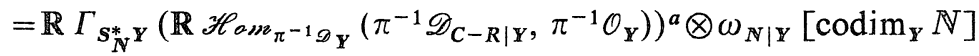

$$
\begin{aligned}
& =\mathbb{R} \mathscr{H}_{a m_{\pi^{-1} \mathscr{D}_{\mathbf{Y}}}}\left(\mathscr{D}_{C-R \mid \mathbf{Y}}, \mathbb{R} \Gamma_{S_{N}^{*} \mathbf{Y}}\left(\pi^{-1} \mathcal{O}_{\mathbf{Y}}\right)^{a}\right) \otimes \omega_{N \mid \mathbf{Y}}\left[\operatorname{codim}_{\mathbf{Y}} \mathbb{N}\right] \\
& =\mathbb{R} \mathscr{H}_{a m_{\pi^{-1} \mathscr{R}_{Y}}}\left(\mathscr{D}_{C-R \mid Y}, \mathscr{C}_{N}\right) \\
& =\mathbb{R} \mathscr{H}_{a m_{\mathscr{G}_{Y}}}\left(\mathscr{D}_{\mathrm{C}-R \mid \mathbf{Y}}, \mathscr{C}_{N}\right) \text {. }
\end{aligned}
$$

Dans le cas particulier (i.e. l'exemple 1.2.3 et l'exemple 1.2.4), M. Kashiwara-T. Kawai [18] ont obtenu cette relation microlocale en utilisant la théorie du système elliptique.

Remarquons que le point essentiel pour la démonstration du théorème 1.2.2 est la condition " $\phi: \mathbb{Y} \rightarrow \mathbb{X}$ est une submersion". En utilisant cette condition, on peut aussi déduire du résultat de M. Kashiwara-T. Kawai [18] notre résultat.

Exemple 1.2.3. Soit $\mathbb{R}$ une variété analytique réelle de complexifiée $\mathbb{X}$. D'après le lemme 1.1.6, on a alors

$$
\mathscr{D}_{C-R \mid \mathbf{X}}=\mathscr{D}_{\mathbf{X} \rightarrow \mathbf{X}} \otimes_{\mathscr{D}_{\mathbf{X}}} \mathscr{D}_{\mathbf{X}}=\mathscr{D}_{\mathbf{X}} .
$$

En utilisant le théorème précédent, on retrouve la définition du faisceau des fonctions analytiques réelles, celle des hyperfonctions et celle des microfonctions.

Exemple 1.2.4. Soit $\Omega$ un ouvert de $\mathbb{X}$ dont la frontière $\mathbb{N}=\partial \Omega$ est une hypersurface analytique réelle. On note $\mathbb{F}$ le complement de $\Omega$ dans $\mathbb{X}$. On note $j$ l'inclusion de $\Omega$ dans $\mathbb{X}$. On a alors les suites exactes des faisceau sur $\mathbb{N}$ (cf. §3):

$$
\begin{aligned}
& 0 \longrightarrow \mathcal{O}_{\mathbf{X}} \longrightarrow \mathcal{j}\left(\dot{j}^{-1} \mathcal{O}_{\mathbf{X}}\right) \longrightarrow \mathscr{H}_{\mathbf{F}}^{1}\left(\mathcal{O}_{\mathbf{X}}\right) \longrightarrow 0 \\
& 0 \longrightarrow \mathbb{R}^{k} \mathscr{J}^{*}\left(j^{-1} \mathcal{O}_{\mathbf{X}}\right) \longrightarrow \mathscr{H}_{\mathbf{F}}^{k+1}\left(\mathcal{O}_{\mathbf{X}}\right) \longrightarrow 0 \quad k \geqq 1 .
\end{aligned}
$$

On désignera par $\mathbb{N}_{+}$le fibré conormal extérieur au bord $\mathbb{N}=\partial \Omega$ en sphères. On a les isomorphismes suivants:

$$
\mathscr{H}_{F}^{k}\left(\mathcal{O}_{\mathbf{X}}\right) \mid \mathbb{N}=\pi_{N \mid \mathbb{X}^{*}}\left(\mathscr{H}_{S_{N}^{*} X^{*}}^{k}\left(\pi_{N \mid X}^{-1}\left(\mathcal{O}_{\mathbf{X}}\right)\right)^{a} \mid \mathbb{N}_{+}\right)
$$


En appliquant le résultat précédent à cette situation, on retrouve les isomorphismes suivants:

$$
\begin{aligned}
& \left(\left(\not{j} * j^{-1} \mathcal{O}_{\mathbf{X}}\right) / \mathcal{O}_{\mathbf{X}}\right) \mid \mathbb{N}=\pi_{N \mid \mathbf{X}^{*}}\left(\mathscr{H}_{a m_{\mathscr{E}_{\mathbf{Y}}}}\left(\mathscr{D}_{C-R \mid \mathbf{Y}}, \mathscr{C}_{\boldsymbol{N}}\right) \mid \mathbb{N}_{+}\right),
\end{aligned}
$$

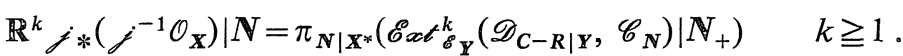

Exemple 1.2.5 (H. Lewy [30], cf. [4] et S-K-K. Chap. 1, Ex. 3.2.4). Plaçons-nous dans $\boldsymbol{X}=C^{2}$ munie des coordonnées $\left(z_{1}, z_{2}\right)=\left(x_{1}+i y_{1}, x_{2}+i y_{2}\right)$, et posons:

$$
\Omega=\left\{\left(z_{1}, z_{2}\right) \mid x_{2}+x_{1}^{2}+y_{1}^{2}<0\right\}, j: \Omega \longrightarrow \mathbb{X}, \quad \mathbb{N}=\partial \Omega .
$$

Soit $W$ l'espace complexe $C^{3}$ muni des coordonnées $\left(w_{1}, w_{2}, w_{3}\right)=\left(u_{1}+i v_{1}\right.$, $\left.u_{2}+i v_{2}, u_{3}+i v_{3}\right)$. On note $(u ; \eta)$ le système des coordonnées symplectique sur $\mathbb{T}_{R^{3}}^{*} W$. On définit l'application $\phi: \mathbb{W}$ par:

$$
\phi\left(w_{1}, w_{2}, w_{3}\right)=\left(w_{1}+i w_{2},-w_{1}^{2}-w_{2}^{2}+i w_{3}\right) .
$$

Il est facile de voir que $\phi\left(R^{3}\right)=\mathbb{N}$ et que

$$
\phi^{*}\left(\mathscr{D}_{C-R}\right)=\phi^{*}\left(\mathscr{D}_{C-R \mid \mathbf{Y}}\right)=\mathscr{D}_{W} / \mathscr{D}_{W} P, \quad \phi^{*}\left(\mathbb{T}_{\mathbf{N}}^{*} \mathbf{X}\right)=S S\left(\mathscr{D}_{W} / \mathscr{D}_{W} P\right) \cap \mathbb{T}_{R^{3}}^{*} W,
$$

où

$$
P=\frac{1}{2}\left(\frac{\partial}{\partial w_{1}}+i \frac{\partial}{\partial w_{2}}\right)-i\left(w_{1}+i w_{2}\right) \frac{\partial}{\partial w_{3}} .
$$

De plus par cette identification $\phi^{*}$, le fibré conormal extétieur au bord $\mathbb{N}$ correspond à l'ensemble suivant:

$$
\left\{(u ; \eta) \mid \eta_{1}+u_{1} \eta_{3}=0, \eta_{2}-u_{2} \eta_{3}=0, \eta_{3}>0\right\} .
$$

On désigne par $R_{+}^{3}$ l'ensemble précédent (i.e. $R_{+}^{3}=\phi^{*}\left(\boldsymbol{N}_{+}\right)$). On a alors:

$$
\begin{aligned}
& \mathscr{H}_{a m_{\mathscr{E}} W}\left(\mathscr{D}_{W} \mid \mathscr{D}_{W} P, \mathscr{C}_{R^{3}}\right) \mid R_{+}^{3}=\phi^{*}\left(\left(j_{*}\left(j^{-1} \mathcal{O}_{\mathbf{x}}\right) / \mathcal{O}_{\mathbf{X}}\right) \mid \mathbb{N}\right) \text {. }
\end{aligned}
$$

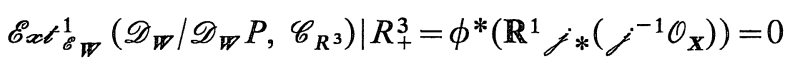

(voir le théorème 2.2.3).

Corollaire 1.2.6 (cf. T. Kawai [25]). Soit X une variété analytique complexe. Soit $\mathbf{N}$ une sous-variété analytique réelle de $\mathbb{X}$. Si $\mathbb{N}$ est générique, on $a$

$$
\begin{aligned}
& \mathscr{H}_{\boldsymbol{N}}^{k}\left(\mathcal{O}_{\mathbf{X}}\right)=0, \quad \mathscr{H}_{S_{N}^{*} \mathbf{X}}^{k}\left(\pi_{\boldsymbol{N} \mid \mathbf{X}}{ }^{-1} \mathcal{O}_{\mathbf{X}}\right)=0 \\
& \quad \text { pour } k<\operatorname{codim}_{\mathbf{X}} N, \operatorname{dim} \mathbf{X}-\operatorname{codim}_{\mathbf{X}} \mathbb{N}<k .
\end{aligned}
$$

Démonstration. On a $\operatorname{dim} \operatorname{proj}\left(\mathscr{D}_{C-R \mid \boldsymbol{Y}}\right)=\operatorname{dim} \mathbb{X}-\operatorname{codim}_{\mathbf{X}} \mathbb{N}$. On en déduit le résultat facilement. 


\section{§2. Analyse Microlocale de la Variété de Cauchy-Riemann.}

A) Nous allons étudier la structure microlocale de la variété générique dans la variété complexe, en utilisant la forme de Levi généralisée de I. Naruki [33]. Nous démontrerons aussi que la forme de Levi généralisée de I. Naruki coïncide avec celle de $\mathrm{S}-\mathrm{K}-\mathrm{K}$ pour le système des équations de Cauchy-Riemann tangentielles.

Dans tout ce paragraphe, $\mathbb{X}$ désignera une variété analytique complexe de dimension $m$. On identifiera souvent $\mathbb{T X}_{R}\left(\right.$ resp. $\left.\mathbb{T}^{*} \mathbb{X}_{R}\right)$ à $\mathbb{T} \mathbb{X}\left(\right.$ resp. $\left.\mathbb{T}^{*} \mathbb{X}\right)$. Si $f$ est une fonction de classe $C^{1}$ sur $\mathbb{X}_{R}, d f(p) \in \mathbb{T}_{p}^{*} \mathbb{X}_{R}$ correspondant à $\partial f(p)$ $\in$ T⿱ $_{p}^{*} \mathbb{X}$ (où $d=\partial+\bar{\partial}$ ) par cette identification.

Soit $\mathbb{N}$ une sous-variete analytique réelle de dimension $n$. Supposons que $\mathbb{N}$ soit définie localement par les équations à valeurs réelles:

$$
f_{1}(z, \bar{z})=f_{2}(z, \bar{z})=\cdots=f_{2 m-n}(z, \bar{z})=0
$$

avec

$$
d f_{1}(z, \bar{z}) \wedge d f_{2}(z, \bar{z}) \wedge \cdots \wedge d f_{2 m-n}(z, \bar{z}) \neq 0 \quad \text { au voisinage de } \quad p \in \mathbb{N} .
$$

On désigne par $\mathbb{H} T(\mathbb{N})_{p}$ l'espace vectoriel cornplexe tangent à $\mathbb{N}$ en $p$, i.e.

$$
\begin{array}{r}
H \mathbb{H}(\mathbb{N})_{p}=\left\{w \frac{\partial}{\partial z} \in \mathbb{T}_{p} \mathbb{X} \mid\left\langle w \frac{\partial}{\partial z}, \alpha_{1} \partial f_{1}+\cdots+\alpha_{2 m-n} \partial f_{2 m-n}\right\rangle(p)=0\right. \\
\left.\forall\left(\alpha_{1}, \alpha_{2}, \ldots, \alpha_{2 m-n}\right) \in C^{2 m-n}\right\} .
\end{array}
$$

Si $\mathbb{N}$ est générique dans $\mathbb{X}$, on a $\operatorname{dim}_{C} \mathbb{H} \mathbb{T}(\mathbb{N})_{p}=n-m$.

Rappelons d'abord la

Définition 2.1. 1 (I. Naruki [33]). Dans la situation précédente, supposons que $\mathbb{N}$ soit générique dans $\mathbb{X}$. Fixons maintenant $p \in \mathbb{N}$ et $q \in\left(\mathbb{T}_{\mathbb{N}}^{*}\right)_{p}$ avec $q=\left(k_{1} d f_{1}+k_{2} d f_{2}+\cdots+k_{2 m-n} d f_{2 m-n}\right)(p)$ où $k_{j} \in C$. On pose

$$
\left\{\mathscr{L}_{N}(f, q)\right\}(w, \bar{w})=\left\{\partial \bar{\partial}\left(k_{1} f_{1}+k_{2} f_{2}+\cdots+k_{2 m-n} f_{2 m-n}\right)(p)\right\}(w, \bar{w}) \text { où } w \in \mathbb{H} \mathbb{T}(\mathbb{N})_{p} .
$$

On l'appelle la forme de Levi généralisée de $\left(f_{1}, f_{2}, \ldots, f_{2 m-n}\right)$ au point $q \in$ $\left(\mathbb{T}_{N}^{*} \mathbb{X}\right)_{p}$. Il est facile de voir que la signature de la forme de Levi généralisée de $\left(f_{1}, f_{2}, \ldots, f_{2 m-n}\right)$ ne depend que de $\mathbb{N}$ et de $q \in\left(\mathbb{T} * *_{N} \mathbb{X}\right)_{p}$.

Remarquons que si $\mathbb{N}$ est une hypersurface définie par une équation réelle $f(z, \bar{z})=0$ au voisinage de $p \in \mathbb{N}$, on a 


$$
\mathscr{L}_{\mathbf{N}}(f, k d f(p))=k \partial \bar{\partial} f(p)
$$

Remarquons aussi que si $\boldsymbol{X}$ est un complexifié de $\boldsymbol{M}$, on a $\boldsymbol{H} \boldsymbol{T}(\boldsymbol{M})=\mathbf{T}_{\boldsymbol{M}} \boldsymbol{M}$ et que $\mathscr{L}_{M}=0$.

On pose $\omega$ la 1 -forme canonique et $\sigma$ la 2 -forme symplectique sur $\mathbf{X}$ :

$$
\omega=\sum_{j=1}^{m} \zeta_{j} d z_{j}, \sigma=d \omega=\sum_{j=1}^{m} d \zeta_{j} \wedge d z_{j}
$$

Rappelons ensuite la

Définition 2.1.2 (P. Schapira [38]). Soit $\boldsymbol{\Lambda}$ une partie de $\boldsymbol{T}^{*} \boldsymbol{X}$.

(1) On dit que $\Lambda$ est une variété $R$-lagrangienne si $\Lambda$ est une variété analytique réelle, conique, lagrangienne dans $\boldsymbol{T}^{*}\left(\boldsymbol{X}_{R}\right)$.

(2) Soit $\mathbb{A}$ une variété $R$-lagrangienne. On dit qu'elle est I-symplectique si la restriction de $\operatorname{Im} \sigma$ (la partie imaginaire de $\sigma$ ) à $\mathbb{\Lambda}$ est non dégénérée.

$P$. Schapira [38] a remarqué le

Lemme 2.1.3. Soit $\mathbb{1}$ une variété $R$-lagrangienne. Pour que $\mathbb{A}$ soit I-symplectique, il faut et il suffit que $\mathbb{T}^{*} \boldsymbol{X}$ s'identifie à un complexifié de $\mathbb{\Lambda}$.

En utilisant le résultat précédent, nous obtenons la

Proposition 2.1.4. Soit $\mathbf{N}$ une sous-variété analytique réelle de $\mathbf{X} . \quad T_{N}^{*} \boldsymbol{X}$ est I-symplectique au voisinage de $q \in\left(\mathbb{T}_{\mathbf{N}}^{*} \mathbf{X}\right)_{p}$ si et seulement si:

i) $\mathbf{N}$ est générique dans $\boldsymbol{X}$ et que

ii) La forme de Levi généralisée de $\mathbf{N}$ au point q est non dégénérée.

Dans la suite, $\boldsymbol{N}$ désignera une sous-variété analytique réelle, générique de $\boldsymbol{X}$. Nous supposons aussi que $\mathbb{N}$ soit définie localement par les équations $f_{1}=f_{2}=\cdots=f_{2 m-n}=0$, où $f_{j}$ désignent des fonctions analytiques réelles aux valeurs réelles.

Soit $(z, w)$ un système de coordonnées locales de $\boldsymbol{X} \times \overline{\boldsymbol{X}}$. On note $f_{j}(z, w)$ les complexifiés des $f_{j}$ dans $\mathbf{X} \times \overline{\mathbf{X}}$. Le complexifié de $\boldsymbol{N}$ dans $\boldsymbol{X} \times \overline{\boldsymbol{X}}$, noté $\boldsymbol{Y}$, est défini par:

$$
f_{1}(z, w)=f_{2}(z, w)=\cdots=f_{2 m-n}(z, w)=0 .
$$

$\mathbf{N}$ étant générique, on peut supposer que la condition suivante soit satisfaite:

$$
\operatorname{det}\left(\frac{\partial\left(f_{1}, f_{2}, \ldots, f_{2 m-n}\right)}{\partial\left(w_{n-m+1}, w_{n-m+2}, \ldots, w_{m}\right)}\right) \neq 0 \quad \text { sur } \mathbb{Y}
$$

Introduisons les opérateurs différentiels 


$$
X_{\bar{J}}=\frac{\partial}{\partial w_{j}}-\sum_{h=n-m+1}^{m} a_{\bar{J}}^{h}(z, w) \frac{\partial}{\partial w_{h}} \quad j=1,2, \ldots, n-m .
$$

de sorte à satisfaire les conditions $X_{\bar{J}}\left(f_{k}\right)=0, k=1,2, \ldots, 2 m-n$;

$X_{j}$ sont uniquement déterminés par ces conditions. Il est facile de voir que $X_{\bar{J}}$ sont des opérateurs différentiels sur $\mathbb{Y}$ (cf. B. Malgrange, Analytic spaces, L'Enseignement mathém., t. XIV, fasc. I pp.1-28). Et que l'on obtient tout de suite le

Lemme 2.1.5. On pose $\mathscr{Q}_{\mathbf{Y}}=\mathscr{D}_{\mathbf{Y}} \mathbb{X}_{\overline{1}}+\mathscr{D}_{\mathbf{Y}} \mathbb{X}_{\overline{2}}+\cdots+\mathscr{D}_{\mathbf{Y}} \mathbb{X}_{\overline{n-m}}$. La suite de $\mathscr{D}_{\mathbf{Y}}$-modules suivante est exacte:

$$
0 \longleftarrow \mathscr{D}_{C-R \mid \boldsymbol{Y}} \longleftarrow \mathscr{D}_{\mathbf{Y}} \longleftarrow \mathscr{Q}_{\mathbf{Y}} \longleftarrow 0 .
$$

Rappelons la définition de la forme de Levi au sens du S-K-K.

Définition 2.1.6 (S-K-K. Chap. 3, Def. 2.3.1). Soit $V$ un sous ensemble analytique involutif de $\mathbb{P}^{*} \mathbb{Y}, \mathbb{Y}$ désignant un complexifié de $\mathbb{N}$. Supposons que $\mathbb{V}$ soit défini localement par les équations $p_{1}=p_{2}=\cdots=p_{d}=0$ au voisinage de $q \in \mathbb{S}_{N}^{*} \mathbb{Y}$. Alors la forme de Levi généralisée de $\mathbb{V}$ est par définition la forme hermitienne dont les coefficients sont les crochets de Poisson

$$
\left\{p_{j}, p_{k}^{C}\right\}(q)_{\substack{1 \leqq j \leqq d \\ 1 \leqq k \leqq d}}, \quad \text { où on a posé } p_{k}^{C}=\bar{p}_{k} .
$$

Nous obtenons alors la

Proposition 2.1.7. Soit $\mathbb{N}$ une sous-variété analytique réelle de dimension $n$ dans une variété complexe $\mathbb{X}$ de dimension $m$. Si $\mathbb{N}$ est générique dans $\mathbb{X}$, alors la signature de la forme de Levi-Naruki de $\mathbb{N}$ est égale à celle de $S-K-K$ pour le système des équations de Cauchy-Riemann tangentielles sur $\mathbb{N}$.

Démonstration. Choisissons les équations locales de $\mathbb{N}$ de façon à satisfaire les conditions suivantes:

$$
\partial_{z_{h}} f(p)=0, \quad h=1,2, \ldots, n-m, \quad f={ }^{t}\left(f_{1}, f_{2}, \ldots, f_{2 m-n}\right) .
$$

On pose:

$D=\operatorname{det}\left(\partial_{z_{n-m+1}} f, \partial_{z_{n-m+2}} f, \ldots, \partial_{z_{m}} f\right), \quad \bar{D}=\operatorname{det}\left(\partial_{\bar{z}_{n-m+1}} f, \partial_{\bar{z}_{n-m+2}} f, \ldots, \partial_{\bar{z}_{m}} f\right)$, et

$$
\begin{array}{ll}
E_{\alpha, j}=\operatorname{det}\left(\partial_{z_{n-m+1}} f, \ldots, \stackrel{\alpha}{\partial}_{z_{j}} f, \ldots, \partial_{z_{m}} f\right), & n-m+1 \leqq \alpha \leqq m \\
\bar{E}_{\alpha, j}=\operatorname{det}\left(\partial_{\bar{z}_{n-m+1}} f, \ldots,{\stackrel{\alpha}{\partial_{\bar{z}}}}_{\bar{z}_{j}} f, \ldots, \partial_{\bar{z}_{m}} f\right) & 1 \leqq j \leqq n-m .
\end{array}
$$

On a alors 


$$
\begin{array}{r}
X_{j}=\frac{\partial}{\partial z_{j}}-\sum_{\alpha=n-m+1}^{m} \frac{E_{\alpha, j}}{D} \frac{\partial}{\partial z_{\alpha}}, \quad X_{\bar{k}}=\frac{\partial}{\partial \bar{z}_{k}}-\sum_{\beta=n-m+1}^{m} \frac{\bar{E}_{\beta, \bar{k}}}{\bar{D}} \frac{\partial}{\partial \bar{z}_{\beta}}, \\
1 \leqq j, k \leqq n-m .
\end{array}
$$

$\boldsymbol{X}_{j}$ désigne le conjugué complexe de $X_{\bar{J}}$ sur $\boldsymbol{N}$. On obtient que

$$
\begin{aligned}
{\left[X_{j}, X_{\bar{k}}\right]=} & \sum_{\alpha=n-m+1}^{m}\left\{\partial_{\bar{z}_{k}}\left(\frac{E_{\alpha, j}}{D}\right) \partial_{z_{\alpha}}-\partial_{z_{j}}\left(\frac{\bar{E}_{\alpha, \bar{k}}}{\bar{D}}\right) \partial_{\bar{z}_{\alpha}}\right\} \\
& +\sum_{\alpha, \beta=n-m+1}^{m}\left\{\frac{E_{\alpha, j}}{D} \partial_{z_{\alpha}}\left(\frac{\bar{E}_{\beta, \bar{k}}}{\bar{D}}\right) \partial_{\bar{z}_{\beta}}-\frac{\bar{E}_{\beta, \bar{k}}}{\bar{D}} \partial_{\bar{z}_{\beta}}\left(\frac{E_{\alpha, j}}{D}\right) \partial_{z_{\alpha}}\right\} .
\end{aligned}
$$

Introduisons une relation $\equiv$ entre les opérateurs d'ordre inférieur ou égale à 1 au point $p \in \mathbb{N}$ comme ci-desous:

$$
P \equiv Q \Longleftrightarrow\left\{\sigma_{1}(P-Q)\right\}\left(\pi^{-1}(p)\right)=0
$$

où $\sigma_{1}(P-Q)$ désigne la partie principale de $P-Q$. Alors

$$
\begin{aligned}
{\left[X_{j}, X_{\bar{k}}\right] } & \equiv \sum_{\alpha=n-m+1}^{m}\left\{\partial_{\bar{z}_{k}}\left(\frac{E_{\alpha}, j}{D}\right) \partial_{z_{\alpha}}-\partial_{z_{j}}\left(\frac{\bar{E}_{\alpha, \bar{k}}}{\bar{D}}\right) \partial_{\bar{z}_{\alpha}}\right\} \\
& \equiv \frac{1}{D \bar{D}} \sum_{\alpha=n-m+1}^{m}\left\{\bar{D} \partial_{\bar{z} k}\left(E_{\alpha, j}\right) \partial_{z_{\alpha}}-D \partial_{z_{j}}\left(\bar{E}_{\alpha, \bar{k}}\right) \partial_{\bar{z}_{\alpha}}\right\} \\
& \equiv \frac{1}{D \bar{D}} \sum_{\alpha=n-m+1}^{m}\left\{\bar{D} E_{\alpha, j, \bar{k}} \partial_{z_{\alpha}}-D \bar{E}_{\alpha, \bar{k}, j} \partial_{\bar{z}_{\alpha}}\right\}
\end{aligned}
$$

où on a pose

$$
\begin{aligned}
& E_{\alpha, j, \bar{k}}=\operatorname{det}\left(\partial_{z_{n-m+1}} f, \ldots, \partial_{z_{j}} \stackrel{\alpha}{\partial}_{\bar{z}_{k}} f, \ldots, \partial_{z_{m}} f\right) \\
& \bar{E}_{\alpha, \bar{k}, j}=\operatorname{det}\left(\partial_{\bar{z}_{n-m+1}} f, \ldots, \partial_{\bar{z}_{k}}{\stackrel{\alpha}{z_{j}}}_{z_{j}} f, \ldots, \partial_{\bar{z}_{m}} f\right) \quad 1 \leqq j, k \leqq n-m .
\end{aligned}
$$

D'autre part on a

$$
\left\{\sigma_{1}\left(X_{j}\right), \sigma_{1}\left(X_{\bar{k}}\right)\right\}=\sigma_{1}\left(\left[X_{j}, X_{\bar{k}}\right]\right) .
$$

On en déduit le résultat facilement.

c. q.f.d.

B) On étudie ici le faisceau des solutions microfonctions du système des équations de Cauchy-Riemann tangentielles sur la sous-variété générique de codimension quelconque.

I. Naruki [33] a sérieusement étudié ce problème dans le cadre $C^{\infty}$. En utilisant les théorèmes de structure de $\mathrm{S}-\mathrm{K}-\mathrm{K}$, on retrouve le résultat de $\mathrm{I}$. Naruki du point de vue microlocal.

Remarquon que R. O. Wells, Jr [44] a traité la variété de Cauchy-Riemann pour l'étude de la transformation de Penrose. 
Rappelons d'abord le

Théorème 2.2.1 (S-K-K. Chap. 3, Th. 2.3.10). Soit $\mathscr{A l}$ un système d'opérateurs pseudo-différentiels défini sur une ouvert $\mathbb{U}$ de $\mathbb{P} * \mathbb{X}$. Supposons que le support $V(=S S(\mathscr{M}))$ du système soit défini localement par les équations

$$
p_{1}(z, \zeta)=p_{2}(z, \zeta)=\cdots=p_{d}(z, \zeta)=0
$$

(1) Supposons que la forme de Levi de $V$ ait au moins q valeurs propres strictement négatives au voisinage $\omega$ de $v^{-1}(z, \zeta)$. On a

$$
\mathscr{E}_{x x t}^{j}\left(\mathscr{E}, \mathscr{M}_{\mathbf{X}}, \mathscr{C}_{M}\right)_{\omega}=0 \quad \text { pour } j<q \text {. }
$$

$v$ désigne l'application naturelle $v: \mathbb{S}_{\mathbb{M}}^{*} \mathbb{X} \rightarrow \mathbb{P}^{*} \mathbb{X}$.

(2) Supposons que la forme de Levi de $\mathbb{V}$ ait au moins $p$ valeurs propres strictement positives au voisinage $\omega$ de $v^{-1}(z, \zeta)$. On a

$$
\begin{aligned}
\mathscr{E}_{x t_{\mathscr{E}_{X}, \boldsymbol{Z}}^{j}}^{j}\left(\mathscr{M}, \mathscr{C}_{M}\right)=0 \quad & \text { pour } j>\sup _{(x, \zeta) \in \omega} \operatorname{dim}_{\operatorname{moj}} \operatorname{proj}_{(x, \xi)} \mathscr{M}-p, \\
& \text { pour tout ensemble fermé } \mathbb{Z} \text { de } \omega .
\end{aligned}
$$

Nous obtenons enfin le

Thèoréme 2.2.2 (1. Naruki [33]). Soit $\mathbb{N}$ une sous-variété analytique réelle, générique de $\mathbb{X}$. Soit $\mathbb{Y}$ un complexifié de $\mathbb{N}$ dans $\mathbb{X} \times \overline{\mathbf{X}}$. On désigne par $\mathscr{D}_{C-R \mid Y}$ le système des équations de Cauchy-Riemann tangentielles sur $\mathbb{N}$.

(1) Si la forme de Levi-Naruki de $\mathbb{N}$ a au moins $q$ valeurs propres strictement négatives au voisinage de $q^{0} \in S_{N}^{*} \mathbb{X}$, on a

$$
\mathscr{E}_{x \in t_{\mathbf{Y}}}^{j}\left(\mathscr{D}_{C-R \mid \mathbf{Y}}, \mathscr{C}_{N}\right)_{q^{0}}=0 \quad \text { pour } j<q .
$$

(2) Si la forme de Levi-Naruki de $\mathbb{N}$ a au moins $p$ valeurs propres strictement positives au voisinage de $q^{0} \in \mathbb{S}_{\mathbb{N}}^{*} \mathbb{X}$, on a

$$
\mathscr{E}_{x t_{\mathscr{E}_{Y}}^{j}}^{j}\left(\mathscr{D}_{C-R \mid \boldsymbol{Y}}, \mathscr{C}_{N}\right)_{q^{0}}=0 \quad \text { puor } j>\operatorname{dim}_{R} \mathbb{N}-\operatorname{dim}_{C} \mathbb{X}-p .
$$

Démonstration. La proposition 2.1.7 montre que la signature de la forme de Levi-Naruki de $\mathbb{N}$ coïncide avec celle de $\mathscr{D}_{C-R \mid \boldsymbol{Y}}$. En appliquant le théorème 2.2.1 à notre situation, nous obtenons le résultat tout de suite.

(2) Il est facile de vérifier que la dimension projective de $\mathscr{D}_{C-R \mid Y}$ est égale à $\operatorname{dim}_{R} \mathbb{N}-\operatorname{dim}_{C} \mathbb{X}$. On en déduit le résultat.

c. q.f.d.

$\mathrm{D}$ 'après le théorème 1.2 .2 , on peut interpréter le théorème 2.2 .2 comme une 
généralisation d'un résultat de $\mathrm{A}$. Andreotti - H. Grauert [2] à la sous-variété générique de codimension quelconque.

Supposons maintenant que $\mathbb{T}_{N}^{*} \mathbf{X}-\mathbb{T}_{N}^{*} \mathbb{N}$ soit une variété I-symplectique au voisinage de $q^{0}$. D'après la proposition 2.1.4, la forme de Levi-Naruki de $\mathbf{N}$ est non dégénérée en $q^{0}$. On déduit du théorème 2.2 .2 que $\mathbb{R} \mathscr{H}_{a m_{\mathscr{E}_{Y}}}\left(\mathscr{D}_{C-R \mid \mathbf{Y}}\right.$, $\mathscr{C}_{N}$ ) est concentré dans un dégré. Plus précisément on a le

Théorème 2.2.3. Soit $T_{N}^{*} \mathbb{X}-T_{N}^{*} \mathbb{X}$ une variété I-symplectique au voisinage $\omega$ de $q^{0}$. Supposons que la signature de la forme de Levi-Naruki de $\mathbb{N}$ soit $\left(\operatorname{dim}_{R} \mathbb{N}-\operatorname{dim}_{C} \mathbb{X}-p, p\right)$ sur $\omega$. On a alors

$$
\begin{aligned}
& \mathscr{E}_{x t_{\mathscr{E}_{Y}}^{j}}^{j}\left(\mathscr{D}_{C-R \mid \mathbf{Y}}, \mathscr{C}_{N}\right)=0 \quad \text { pour } j \neq p, \\
& \mathscr{E}_{x \in t_{\mathscr{E}_{Y}}^{p}}\left(\mathscr{D}_{C-R \mid Y}, \mathscr{C}_{N}\right) \cong \mathscr{C}_{R^{m}}
\end{aligned}
$$

Démonstration. Le système $\mathscr{D}_{C-R \mid \boldsymbol{Y}}$ coïncide microlocalement avec le système de Lewy-Mizohata de la signature $\left(\operatorname{dim}_{R} \mathbb{N}-\operatorname{dim}_{C} \mathbb{X}-p, p\right)$. Le résultat de S-K-K (Chap. 3, Th. 2.3.6) entraîne ce résultat.

c. q.f.d.

\section{§3. Problème de Prolongement et Système Tangentiel.}

Nous envisagerons le problème du prolongement des solutions holomorphes du système d'équations aux dérivées partielles.

Soit $\boldsymbol{X}$ une variété analytique complexe. Soit $\Omega$ un ouvert de $\mathbf{X}$ dont la frontière $N=\partial \Omega$ est de classe $C^{1}$. On notera $j$ l'inclusion naturelle $\Omega \rightarrow \mathbb{X}$. On désigne par $\mathbb{F}$ le complement de $\Omega$ dans $\boldsymbol{X}$.

Etant donné un $\mathscr{D}_{\mathrm{X}}$-module cohérent $\mathscr{M}$ sur $\mathbb{X}$, le faisceau $\mathscr{F}=$ $\mathscr{H}_{\text {am }_{\mathscr{G}_{\mathbf{X}}}}\left(\mathscr{M}, \mathcal{O}_{\mathbf{X}}\right)$ est le faisceau des solutions holomorphes du système $\mathscr{M}$. En désignant par $\mathbb{R}^{q} \mathcal{J}_{*}$ le $q$-ième foncteur dérivé du foncteur $\mathcal{j}_{*}$, on a alors la suite exacte des faisceaux:

$$
\begin{aligned}
& 0 \longrightarrow \mathscr{H}_{F}^{0}(\mathscr{F}) \longrightarrow \mathscr{H}^{0}(\mathscr{F}) \longrightarrow \mathcal{j}^{*}\left(j^{-1} \mathscr{F}\right) \longrightarrow \mathscr{H}_{F}^{1}(\mathscr{F})- \\
& \longrightarrow \mathscr{H}^{1}(\mathscr{F}) \longrightarrow \mathbb{R}^{1} \mathcal{j}^{*}\left(j^{-1} \mathscr{F}\right) \longrightarrow \mathscr{H}_{F}^{2}(\mathscr{F}) \longrightarrow \cdots
\end{aligned}
$$

On obtient donc les suites exactes sur $N$ :

$$
\begin{aligned}
& 0 \longrightarrow \mathscr{F} \longrightarrow j_{*}\left(j^{-1} \mathscr{F}\right) \longrightarrow \mathscr{H}_{F}^{1}(\mathscr{F}) \longrightarrow 0, \\
& 0 \longrightarrow \mathbb{R}^{k} \mathcal{j}_{*}\left(j^{-1} \mathscr{F}\right) \longrightarrow \mathscr{H}_{F}^{k+1}(\mathscr{F}) \longrightarrow 0, \quad k \geqq 1 .
\end{aligned}
$$

On en déduit facilement que tout germe de solutions holomorphes du 
système $\mathscr{M}$ au voisinage du bord sur $\Omega$ (i.e. $\not{j} *\left(\mathcal{J}^{-1} \mathscr{F}\right)$ ) se prolonge à travers le bord si et seulement si $\mathscr{H}_{F}^{1}(\mathscr{F})=0$.

Considérons ensuite la transformation comonoïdale $(\mathbb{X}-\mathbb{N}) \sqcup \mathbb{S}_{N}^{*} \mathbb{X}$ munie de la topologie de co-éclaté. On note $\mathbb{N}_{+}$(resp. $\left.\mathbb{N}_{-}\right)$le fibré conormal extérieur (resp. intérieur) au bord $\mathbb{N}$ en sphères. Nous pouvons identifier $S_{N}^{*} \mathbb{X}$ avec $\mathbb{N}_{+} \sqcup \mathbb{N}_{-}$. Choisissons une demiespace $\Omega \sqcup \mathbb{N}_{-}$de $(\mathbb{X}-\mathbb{N}) \sqcup \mathbb{N}_{+} \sqcup \mathbb{N}_{-}$. On désigne encore par $j$ l'inclusion $j: \Omega \rightarrow \Omega \sqcup \mathbb{N}_{-}$et par $\pi$ la projection naturelle $\Omega \sqcup \mathbb{N}_{-} \rightarrow \mathbb{X}$.

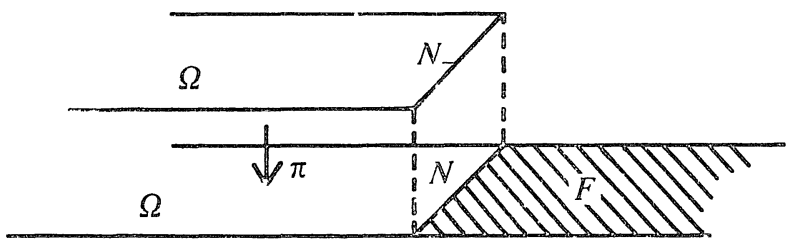

On a les suites exactes des faisceaux sur $\mathbb{N}_{-}$:

$$
\begin{aligned}
& 0 \longrightarrow \pi^{-1} \mathscr{F} \longrightarrow \mathcal{j}^{*}\left(\mathcal{j}^{-1} \pi^{-1} \mathscr{F}\right) \longrightarrow \mathscr{H}_{N_{-}}^{1}\left(\pi^{-1} \mathscr{F}\right) \longrightarrow 0, \\
& 0 \longrightarrow \mathbb{R}^{k} \mathcal{J}^{*}\left(\mathcal{j}^{-1} \pi^{-1} \mathscr{F}\right) \longrightarrow \mathscr{H}_{N-}^{k+1}\left(\pi^{-1} \mathscr{F}\right) \longrightarrow 0, \quad k \geqq 1 .
\end{aligned}
$$

On obtient les isomorphismes naturels suivants:

$$
\mathscr{H}_{F}^{k}(\mathscr{F}) \mid \mathbb{N}=\pi_{N \mid X^{*}}\left(\mathscr{H}_{S_{N}^{*} X^{*}}^{k}\left(\pi_{N \mid X}^{-1} \mathscr{F}\right) \mid \mathbb{N}_{-}\right) \quad \text { quel que soit } k \text {. }
$$

Il est claire d'après ce qui précéde que le problème du prolongement s'interprère par le langage microlocal. Dans le cas où $\mathbb{N}$ est une sous-variété d'une variété $\mathbb{X}$, nous renvoyons à $S-K-K$ (Chap. 1 , Prop. 1.2.3) pour la signification de $\mathbb{R} \Gamma_{S_{N}^{*} X}\left(\pi_{N \mid X}^{-1} \mathscr{F}\right)$.

Puisque nous avons l'intention de traiter le problème du prolongement des faisceaux $\mathscr{E}_{x t_{\mathscr{D}_{\mathbf{X}}}^{k}}\left(\mathscr{M}, \mathcal{O}_{\mathbf{X}}\right)$, nous allons considérer

$$
\mathbb{R} \Gamma_{N} \mathbb{R} \mathscr{H}_{\text {am } \mathscr{D}_{\mathbf{X}}}\left(\mathscr{M}, \mathcal{O}_{\mathbf{X}}\right) \quad \text { et } \quad \mathbb{R} \Gamma_{S_{N}^{*} \mathbb{X}}\left(\pi_{N \mid \mathbf{X}}^{-1} \mathbb{R} \mathscr{H}_{a m_{\mathscr{D}_{X}}}\left(\mathscr{M}, \mathcal{O}_{\mathbf{X}}\right)\right)
$$

dans les catégories dérivées.

Soit $\mathbb{X}$ une variété analytique complexe de complexifiée $\mathbb{X} \times \overline{\mathbb{X}}$. On note $\tau$ la projection naturelle de $\mathbb{X} \times \overline{\mathbb{X}}$ sur $\mathbb{X}$. Etant donné un $\mathscr{D}_{\mathbb{X}}$-module cohérent $\mathscr{M}$, on définit un $\mathscr{D}_{\mathbf{X} \times \overline{\mathbf{X}}}$-module cohérent $\mathscr{M}_{C-R}$ par:

$$
\mathscr{M}_{C-R}=\tau^{*} \mathscr{M}=\mathscr{D}_{\mathbf{X} \times \overline{\mathbf{X}}} \longrightarrow \mathbb{X} \otimes_{\mathscr{D}_{\mathbf{X}}} \mathscr{M}
$$

Ce système coïncide avec le système qui a été introduit par M. Kashiwara 
[16]. Remarquons aussi que T. Kawai [24] et A. Andreotti-M. Nacinovich [5] ont utilisé ce système.

Si on substitue $\mathscr{D}_{\boldsymbol{X}}$ à $\mathscr{M}$, on obtient le système des équations de CauchyRiemann $\mathscr{D}_{C-R}$. Nous allons considérer le système $\mathscr{M}_{C-R}$ dans le cas général. Choisissons d'abord une résolution projective locale de $\mathscr{M}$ :

$$
0 \longleftarrow \mathscr{M} \longleftarrow \mathscr{D}_{X}^{r_{0}} \stackrel{P_{0}}{\longleftarrow} \mathscr{D}_{X}^{r_{1}} \longleftarrow P_{1} \ldots \stackrel{P_{q}}{\longleftarrow} \mathscr{D}_{X}^{r_{q+1}} \longleftarrow 0 .
$$

On désignera par $\widetilde{P}_{j}$ les complexifiés de $P_{j}$ dans $\mathbf{X} \times \overline{\mathbf{X}}$. Il est facile de voir que le complexe suivant est bien défini.

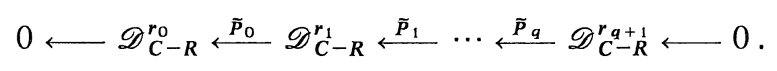

En effet il est facile de voir que

$$
\mathscr{E}_{n d} \mathscr{\mathscr { T }}_{\boldsymbol{X} \times \overline{\boldsymbol{X}}}\left(\mathscr{D}_{C-R}\right)=\widetilde{\mathscr{D}}_{\boldsymbol{X}},
$$

où $\widetilde{\mathscr{D}}_{\boldsymbol{X}}$ désigne le complexifié de $\mathscr{D}_{\mathbf{X}}$ dans $\mathbf{X} \times \overline{\mathbf{X}}$. Il en résulte que le complexe précédent, noté $\mathscr{D}_{C-R}(\mathscr{M})$, est bien défini.

Lemme 3.1.1. Dans la situation précédente, la suite suivante est exacte:

$$
0 \longleftarrow \mathscr{M}_{\mathrm{C}-\mathrm{R}} \longleftarrow \mathscr{D}_{\mathrm{C}-\mathrm{R}}(\mathscr{M}) \text {. }
$$

Démonstration. Par définition on a

$$
\mathscr{D}_{C-R}=\tau^{*} \mathscr{D}_{\boldsymbol{X}} \quad \text { et } \quad \mathscr{M}_{C-R}=\tau^{*} \mathscr{M} .
$$

Rappelons que $\mathscr{D}_{\mathbf{X} \times \overline{\boldsymbol{X}} \rightarrow \boldsymbol{X}}$ est plat sur $\mathscr{D}_{\mathbf{X}}$ (S-K-K. Chap. 2, Lemme 3.5.1). On en déduit le résultat immédiatement.

c. q.f.d.

M. Kashiwara [16] a montré le résultat suivant pour appliquer le résultat de T. Kawai [23] à l'étude du système holonome.

Proposition 3.1.2. Soit $\mathscr{M}$ un $\mathscr{D}_{\mathrm{x}}$-module cohérent. On a alors

$$
\mathbf{R} \mathscr{H}_{a_{m_{X}}}\left(\mathscr{M}, \mathcal{O}_{\mathbf{X}}\right)=\mathbf{R} \mathscr{H}_{a_{m_{X} \times \overline{\mathbf{X}}}}\left(\mathscr{H}_{C-R}, \mathscr{B}_{\boldsymbol{X}_{\boldsymbol{R}}}\right),
$$

où $\boldsymbol{X}_{R}$ désigne la variété analytique réelle sous-jacente à $\mathbf{X}$ et $\mathscr{B}_{\mathbf{X}_{R}}$ désigne le faisceau des hyperfonctions sur $\boldsymbol{X}_{R}$.

Démonstration. La proposition se déduit du lemme 3.1.1.

c. q.f.d.

Ensuite nous allons étudier le module $\mathscr{M}_{C-R \mid \boldsymbol{Y}}$ en supposant que $\mathbb{N}$ soit générique dans $\boldsymbol{X}$. Nous obtenons la

Proposition 3.1.3. Soit $\mathscr{M}$ un $\mathscr{D}_{\mathbf{X}}$-module cohérent. Soient $\mathbb{N}$ une sousvariété analytique réelle de $\boldsymbol{X}$ et $\boldsymbol{Y}$ un complexifié de $\mathbf{N}$ dans $\mathbf{X} \times \overline{\mathbf{X}}$. On 
désigne par $\phi_{C}$ l'inclusion de $\mathbb{Y}$ dans $\mathbb{X} \times \overline{\mathbb{X}}$ et par $\phi$ la composée $\tau \circ \phi_{C}: \mathbb{Y} \rightarrow \mathbb{X}$. Si $\mathbb{N}$ est générique dans $\mathbb{X}$, on obtient alors

(1) $Y$ est non caractéristique par rapport à $\mathscr{M}_{C-R}$,

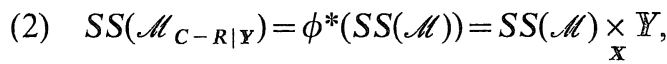

(3) $S S\left(\mathscr{H}_{C-R \mid \mathbb{Y}}\right) \cap \mathbb{T}_{N}^{*} \mathbb{Y}=\phi^{*}\left(S S(\mathscr{U}) \cap \mathbb{T}_{N}^{*} \mathbb{X}\right)$.

Démonstration (1). Le théorème 1.1.1 implique que $\mathbb{Y}$ est non caractéristique par rapport à $\mathscr{D}_{C-R}$. En outre on a $S S\left(\mathscr{M}_{C-R}\right) \subseteq S S\left(\mathscr{D}_{C-R}\right)$. On en déduit que $Y$ est non caractéristique par rapport à $\mathscr{M}_{C-R}$.

(2). Par définitions on a

$$
\mathscr{M}_{C-R \mid Y}=\phi_{C}^{*}\left(\mathscr{M}_{C-R}\right)=\phi_{C}^{*}\left(\tau^{*}(\mathscr{M})\right) .
$$

Le résultat de S-K-K (Chap. 2, Lemme 3.5.1) entraîne que

$$
\mathscr{M}_{C-R \mid Y}=\left(\tau \circ \phi_{C}\right)^{*} \mathscr{M}=\phi^{*} \mathscr{M} \text {. }
$$

Rappelons aussi que $\phi$ est une submersion. Cela termine la démonstration.

(3). On déduit de la proposition 1.2.1 le résultat immédiatement.

c. q.f.d.

L'application $\phi$ étant une submersion, nous allons identifier $\phi^{*}(S S(\mathscr{M})$ $\left.\cap \mathbb{T}_{N}^{*} \mathbb{X}\right)$ avec $S S(\mathscr{M}) \cap \mathbb{T}_{N}^{*} \mathbb{X}$.

Nous obtenons le résultat suivant en utilisant le langage de la théorie des catégories dérivées:

Théorème 3.1.4. Soit $\mathscr{M}$ un $\mathscr{D}_{\mathrm{X}}$-module cohérent. Soient $\mathbb{N}$ une sousvariété analytique réelle de $\mathbb{X}$ et $\mathbb{Y}$ complexifié de $\mathbb{N}$ dans $\mathbb{X} \times \overline{\mathbb{X}}$. Si $\mathbb{N}$ est générique, on a alors les isomorphismes suivants:

(1) $\mathbf{R} \mathscr{H}_{a m_{\mathscr{D}_{\mathbf{Y}}}}\left(\mathscr{M}_{C-R \mid \mathbf{Y}}, \mathscr{H}_{\boldsymbol{N}}\right)=\mathbb{R} \mathscr{H}_{a m_{\mathscr{D}_{\mathbf{X}}}}\left(\mathscr{M}, \mathcal{O}_{\mathbf{X}}\right) \mid \mathbb{N}$,

(2) $\mathbb{R} \mathscr{H}_{a m_{\mathscr{D}_{\mathbf{Y}}}}\left(\mathscr{M}_{C-R \mid \mathbf{Y}}, \mathscr{B}_{N}\right)\left[-\operatorname{codim}_{\mathbf{X}} \mathbb{N}\right]=\mathbb{R} \Gamma_{\boldsymbol{N}} \mathbb{R} \mathscr{H}_{a m_{\mathscr{D}_{X}}}\left(\mathscr{H}_{,} \mathcal{O}_{\mathbf{X}}\right) \otimes \omega_{N \mid \mathbb{X}}$,

(3) $\mathbb{R} \mathscr{H}_{a m_{\mathscr{B}} \mathbf{Y}}\left(\mathscr{M}_{C-R \mid \mathbf{Y}}, \mathscr{C}_{N}\right)\left[-\operatorname{codim}_{\mathbf{X}} \mathbb{N}\right]$

$$
=\mathbb{R} \Gamma_{S_{N}^{*} \mathbb{X}}\left(\pi_{N \mid \mathbf{X}}^{-1}\left(\mathbb{R} \mathscr{H}_{a m_{\mathscr{X}}}\left(\mathscr{M}, \mathcal{O}_{\mathbf{X}}\right)\right)^{a} \otimes \omega_{N \mid \mathbb{X}},\right.
$$

où $\pi_{\boldsymbol{N} \mid \mathbf{X}}$ désigne la projection naturelle $(\mathbb{X}-\mathbb{N}) \sqcup \mathbb{S}_{\mathbb{N}}^{*} \mathbb{X} \rightarrow \mathbb{X}$. Le premier espace étant muni de la topologie de co-éclaté.

En particulier, le problème du prolongement généralisé pour le système $\mathscr{M}$ est équivalent au problème de l'annulation $d u$ faisceau des solutions microfonctions $d u$ système tangentiel $\mathscr{M}_{C-R \mid \mathbf{Y}}$. 
Démonstration (1). $\quad \mathbb{N}$ étant générique, l'application $\phi: \boldsymbol{Y} \rightarrow \boldsymbol{X}$ est une submersion. D'après le théorème 0.1 .4 , on obtient l'isomorphisme suivant:

$$
\mathbf{R} \mathscr{H}_{a m_{\mathscr{D}_{\mathbf{Y}}}}\left(\phi^{*} \mathscr{M}, \mathcal{O}_{\mathbf{Y}}\right)=\phi^{-1}\left(\mathbf{R} \mathscr{H}_{a m_{\mathscr{O}_{X}}}\left(\mathscr{M}, \mathcal{O}_{\mathbf{X}}\right)\right)
$$

i.e.

$$
\mathbb{R} \mathscr{H}_{a m_{\mathscr{O}_{\mathbf{Y}}}}\left(\mathscr{M}_{C-R \mid \mathbf{Y}}, \mathcal{O}_{\mathbf{Y}}\right)=\phi^{-1}\left(\mathbf{R} \mathscr{H}_{a m_{\mathscr{D}_{X}}}\left(\mathscr{M}, \mathcal{O}_{\mathbf{X}}\right)\right) .
$$

Ceci entrîne le résultat tout de suite.

(2). Par le théorème 0.1 .5 on a

$$
\begin{aligned}
& \mathbf{R} \mathscr{H}_{\operatorname{am}_{\mathscr{P}_{\mathbf{Y}}}}\left(\mathscr{M}_{\mathrm{C}-\mathrm{R} \mid \mathbf{Y}}, \mathscr{B}_{\mathbf{N}}\right)\left[-\operatorname{codim}_{\mathbf{X}} \mathbb{N}\right] \\
& =\mathbf{R} \Gamma_{\boldsymbol{N}} \mathbf{R} \mathscr{H}_{a m_{\mathscr{D}} \times \overline{\mathbf{X}}}\left(\mathscr{M}_{C-R}, \mathscr{B}_{\mathbf{x}_{\boldsymbol{R}}}\right) \otimes \omega_{\boldsymbol{N} \mid \mathbf{X}} \cdot
\end{aligned}
$$

En utilisant la proposition 3.1.2 on obtient que

$\mathbb{R} \mathscr{H}_{a m_{\mathscr{P}_{\mathbf{Y}}}}\left(\mathscr{M}_{C-R \mid \boldsymbol{Y}}, \mathscr{B}_{\boldsymbol{N}}\right)\left[-\operatorname{codim}_{\mathbf{X}} N\right]=\mathbb{R} \Gamma_{\boldsymbol{N}} \mathbb{R} \mathscr{H}_{a m_{\mathscr{I}_{\mathbf{X}}}}\left(\mathscr{M}, \mathcal{O}_{\mathbf{X}}\right) \otimes \omega_{N \mid \mathbf{X}}$.

(3) (cf. S-K-K. Chap. 3, Th. 2.2.5).

En utilisant le théorème 1.2.2, on peut démontrer notre résultat. Mais nous donnerons une démonstration directe. On pose $d=\operatorname{codim}_{\mathbf{X}} \mathbb{N}$ et $\mathscr{F}=$ $\mathbb{R} \mathscr{H}_{a_{m_{\mathscr{D}}}}\left(\mathscr{M}, \mathcal{O}_{\mathbf{X}}\right)$. On désigne par $\pi$ la projection $(\boldsymbol{Y}-\boldsymbol{N}) \sqcup \boldsymbol{S}_{\boldsymbol{N}}^{*} \boldsymbol{Y} \rightarrow \boldsymbol{Y}$.

Nous envisageons les triangles suivants avant de démontrer le résultat.

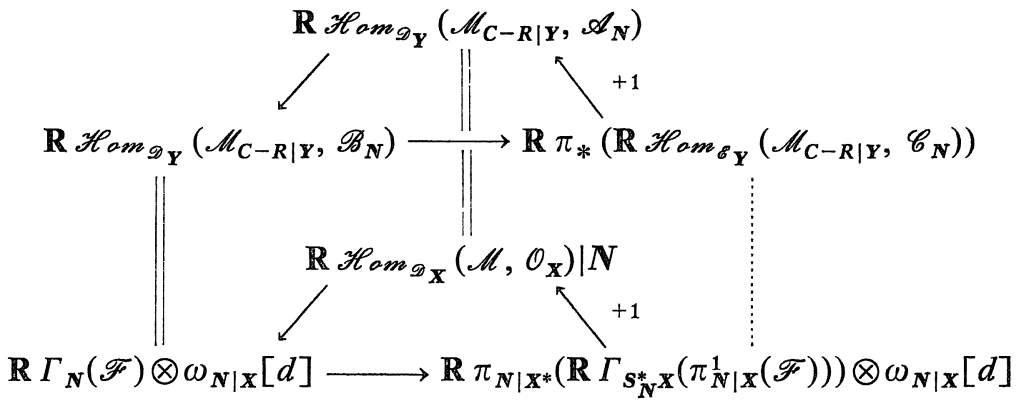

Il résulte alors des résultats précédents qu'il existe un quasi-isomorphisme suivant:

$$
\mathbf{R} \pi_{*}\left(\mathbf{R} \mathscr{H}_{a m_{\mathscr{F}_{\mathbf{Y}}}}\left(\mathscr{M}_{\boldsymbol{C}-\boldsymbol{R} \mid \mathbf{Y}}, \mathscr{C}_{\boldsymbol{N}}\right)\right)=\mathbf{R} \pi_{\boldsymbol{N} \mid \mathbf{X}^{*}}\left(\mathbf{R} \Gamma_{S_{\boldsymbol{N}^{*} \mathbf{X}}}\left(\pi_{\boldsymbol{N} \mid \mathbf{X}}^{-1}(\mathscr{F})\right)^{a}\right) \otimes \omega_{N \mid \mathbf{X}}[d] .
$$

Revenons maintenant à la démonstration. En appliquant un résultat de S-K-K (Chap. 1, Lemme 2.2.3) à cette situation, on obtient l'isomorphisme suivant:

$$
\mathbf{R} \Gamma_{S_{N}^{*} \mathbf{X}}\left(\pi_{\boldsymbol{N} \mid \boldsymbol{X}}^{-1}(\mathscr{F})\right) \otimes \omega_{\boldsymbol{N} \mid \mathbf{X}}[d]=\mathbf{R} \Gamma_{\boldsymbol{S}_{\mathbf{N}}^{*} \mathbf{Y}}\left(\pi^{-1} \phi^{-1}(\mathscr{F})\right) \otimes \omega_{\boldsymbol{N} \mid \mathbf{X}}\left[\operatorname{codim}_{\mathbf{Y}} N\right] .
$$


D'autre part on a

$$
\phi^{-1}(\mathscr{F})=\mathbb{R} \mathscr{H}_{a m_{\mathscr{D}}}\left(\mathscr{M}_{C-R \mid \mathbf{Y}}, \mathcal{O}_{\mathbf{Y}}\right)
$$

Ceci entraîne que

$$
\begin{aligned}
& \mathbb{R} \Gamma_{\boldsymbol{S}_{\boldsymbol{N}}^{*} \mathbf{X}}\left(\pi_{\boldsymbol{N} \mid \boldsymbol{X}}^{-1}(\mathscr{F})\right)^{a} \otimes \omega_{\boldsymbol{N} \mid \boldsymbol{X}}[d] \\
& =\mathbb{R} \Gamma_{S_{N}^{*} \mathbf{Y}}\left(\pi^{-1}\left(\mathbb{R} \mathscr{H}_{\text {am }_{\mathscr{G}} \mathbf{Y}}\left(\mathscr{H}_{C-R \mid \mathbf{Y}}, \mathcal{O}_{\mathbf{Y}}\right)\right)\right)^{a} \otimes \omega_{\mathbb{N} \mid \mathbf{Y}}\left[\operatorname{codim}_{\mathbf{Y}} \mathbb{N}\right] \\
& =\mathbb{R} \Gamma_{S_{N}^{*} \mathbf{Y}}\left(\mathbb{R} \mathscr{H}_{\operatorname{arm}^{-1} \mathscr{D}_{\mathbf{Y}}}\left(\pi^{-1} \mathscr{M}_{C-R \mid \mathbf{Y}}, \pi^{-1} \mathcal{O}_{\mathbf{Y}}\right)\right)^{a} \otimes \omega_{N \mid \mathbf{Y}}\left[\operatorname{codim}_{\mathbf{Y}} \mathbb{N}\right] \\
& =\mathbb{R} \mathscr{H}_{a m_{\pi^{-1} \mathscr{D}_{\mathbf{Y}}}}\left(\mathscr{M}_{C-R \mid \mathbf{Y}}, \mathbb{R} \Gamma_{S_{N}^{*} \mathbf{Y}}\left(\pi^{-1} \mathcal{O}_{\mathbf{Y}}\right)^{a}\right) \otimes \omega_{N \mid \mathbf{Y}}\left[\operatorname{codim}_{\mathbf{Y}} \mathbb{N}\right] \\
& =\mathbb{R} \mathscr{H}_{a m_{\pi^{-1} \mathscr{D}_{Y}}}\left(\mathscr{M}_{C-R \mid \mathbf{Y}}, \mathscr{C}_{N}\right) \\
& =\mathbb{R} \mathscr{H}_{a m_{\mathscr{E}} \boldsymbol{Y}}\left(\mathscr{M}_{C-R \mid \boldsymbol{Y}}, \mathscr{C}_{N}\right) \text {. }
\end{aligned}
$$

\section{Corollaire 3.1.5.}

$$
\begin{aligned}
& \mathscr{H}^{k}\left(\mathbb{R} \Gamma_{\boldsymbol{N}} \mathbb{R} \mathscr{H}_{a m_{\mathscr{O}_{X}}}\left(\mathscr{M}, \mathcal{O}_{\mathbf{X}}\right)\right)=0 \\
& \mathscr{H}^{k}\left(\mathbb{R} \Gamma_{\boldsymbol{S}_{\boldsymbol{N}}^{*} \mathbf{X}}\left(\pi_{\boldsymbol{N} \mid \mathbf{X}}^{-1}\left(\mathbb{R} \mathscr{H}_{a m_{\mathscr{D}_{X}}}\left(\mathscr{M}, \mathcal{O}_{\mathbf{X}}\right)\right)\right)\right)=0
\end{aligned}
$$

pour $k<\operatorname{codim}_{\mathbf{X}} \mathbb{N}$, proj-dim $(\mathscr{M})+\operatorname{dim}_{R} \mathbb{N}-\operatorname{dim}_{C} \mathbb{X}<k$.

Exemple 3.1.6. (Lewy-Mizohata., cf. S-K-K. Chap. 3, §2.3). On pose:

$$
\begin{aligned}
& X=C^{2}=\left\{\left(z_{1}, z_{2}\right) \mid z_{1}=x_{1}+i y_{1}, z_{2}=x_{2}+i y_{2} \in C\right\}, \\
& \Omega=\left\{\left(z_{1}, z_{2}\right) \mid x_{2}+x_{1}^{2}+c y_{1}^{2}<0\right\}, \mathbb{N}=\partial \Omega, \mathscr{H}=\mathscr{D}_{\mathbf{X}} / \mathscr{D}_{\mathbf{X}} \frac{\partial}{\partial z_{1}},
\end{aligned}
$$

et

$$
\begin{aligned}
& W=C^{3}=\left\{\left(w_{1}, w_{2}, w_{3}\right) \mid w_{j} \in C, j=1,2,3\right\}, \\
& \phi\left(w_{1}, w_{2}, w_{3}\right)=\left(w_{1}+i w_{2},-w_{1}^{2}-c w_{2}^{2}+i w_{3}\right), \\
& P_{1}=-\frac{\partial}{\partial w_{1}}-2 i w_{1} \frac{\partial}{\partial w_{3}}, \quad P_{2}=\frac{\partial}{\partial w_{2}}-2 i c w_{2} \frac{\partial}{\partial w_{3}}, \quad \mathscr{N}=\mathscr{D}_{w} / \mathscr{D}_{w} P_{1}+\mathscr{D}_{w} P_{2} .
\end{aligned}
$$

Il est facile de voir que le système $\mathscr{M}_{C-R \mid Y}$ est isomorphe au système $\phi^{*}(\mathscr{H})=\mathscr{N}$ et que

i) Si $c>0$, le système $\mathscr{M}_{C-R \mid Y}$ coïncide avec le système de Lewy-Mizohata de la signature $(2,0)$ sur $S S(\mathscr{M}) \cap \mathbb{N}_{+}$.

ii) Si $c=0$, le système $\mathscr{M}_{C-R \mid \boldsymbol{Y}}$ est un mélange du système de de Rham et du système de Lewy-Mizohata de la signature $(1,0)$ sur $S S(\mathscr{M}) \cap \mathbb{N}_{+}, \mathbb{N}_{+}$désignant le fibré conormal extérieur au bold.

iii) Si $c<0$, le système coïncide avec le système de Lewy-Mizohata de la signature $(1,1)$ sur $S S(\mathscr{M}) \cap \mathbb{N}_{+}$.

Remarquons que l'hypersurface $x_{2}+x_{1}^{2}-y_{1}^{2}=0$ est Levi plat cepandant 
que le système $\mathscr{U}_{C-\boldsymbol{R} \mid \boldsymbol{Y}}$ n'est pas pseudo-convexe au sens de S-K-K (cf. le théorème 4.1.6 et le théorème 4.2.1).

On peut aussi obtenir (comme dans l'exemple 3.1.6) le mélange du système de de Rham, du système de Lewy-Mizohata et du système de Cauchy-Riemann partiel de façon naturelle.

\section{§4. Théorèmes de Prolongement.}

A) Dans ce paragraphe nous donnerons quelques résultats comme applications $\mathrm{du}$ théorème 3.1.4 au problème du prolongement des solutions holomorphes d'équations aux dérivées partielles.

On désigne par $\boldsymbol{N}$ une sous-variété analytique réelle, générique dans une variété analytique complexe $\boldsymbol{X}$. On désigne par $\boldsymbol{Y}$ un complexifié de $\boldsymbol{N}$ dans $\mathbb{X} \times \overline{\mathbf{X}}$. Soit $\mathscr{M}$ un $\mathscr{D}_{\mathbf{X}}$-module cohérent.

Définition 4.1.1. Nous dirons que $\mathbf{N}$ est non caractéristique par rapport à $\mathscr{M}$ si :

$$
S S(\mathscr{M}) \cap \boldsymbol{T}_{\boldsymbol{N}}^{*} \boldsymbol{X} \subseteq \boldsymbol{T}_{N}^{*} \boldsymbol{N}
$$

Remarquons que si $\boldsymbol{N}$ est totalement réelle, la définition précédente coïncide avec la définition 0.1.6. Nous obtenons le

Théorème 4.1.2. Soit $\mathscr{M}$ un $\mathscr{D}_{\mathbf{X}}$-module cohérent. Supposons que $\mathbf{N}$ soit générique dans $\boldsymbol{X}$ et que non caractéristique par rapport à $\mathscr{M}$. On a alors

$$
\begin{aligned}
& \mathbf{R} \mathscr{H}_{a m_{\mathscr{E}_{Y}}}\left(\mathscr{M}_{C-R \mid Y}, \mathscr{C}_{N}\right)=0 \text {, } \\
& \mathbf{R} \mathscr{H}_{a m_{\mathscr{D}} \mathbf{Y}}\left(\mathscr{M}_{C-R \mid \mathbf{Y}}, \mathscr{A}_{N}\right)=\mathbf{R} \mathscr{H}_{a m_{\mathscr{D}_{Y}}}\left(\mathscr{M}_{C-R \mid \mathbf{Y}}, \mathscr{B}_{N}\right) \text {. }
\end{aligned}
$$

Supposons maintenant que $\boldsymbol{N}$ soit totalement réelle. On a alors $\mathscr{M}_{C-R \mid \boldsymbol{Y}}=\mathscr{M}$. Donc dans cette situation le théorème 4.1.2 coïncide avec le résultat de $M$. Sato (le théorème 0.1.7).

Démonstration. D'après la proposition 3.1 .3 , on a

$$
S S\left(\mathscr{M}_{C-R \mid \boldsymbol{Y}}\right) \cap \mathbf{T}_{\boldsymbol{N}}^{*} \boldsymbol{Y}=\phi^{*}\left(S S(\mathscr{U}) \cap \mathbf{T}_{\boldsymbol{N}}^{*} \boldsymbol{X}\right) .
$$

$\boldsymbol{N}$ étant non caractéristique par rapport à $\mathscr{M}$, on obtient que

$$
S S\left(\mathscr{M}_{C-R \mid Y}\right) \cap T_{N}^{*} Y \subseteq T_{N}^{*} N .
$$

Rappelons la définition 0.1.6. Il est évident que le système $\mathscr{M}_{C-R \mid \boldsymbol{Y}}$ est un 
système elliptique sur $\mathbb{N}$. En utilisant le résultat de $M$. Sato (le théorème 0.1 .7 ) on obtient les résultats tout de suite.

c. q.f.d.

Corollaire 4.1.3. Soit $\mathbb{N}$ une sous-variété analytique réelle, générique dans $\mathbb{X}$. Si $\mathbb{N}$ est non caractéristique par rapport à $\mathscr{l l}$, on a alors

$$
\mathbb{R} \Gamma_{\boldsymbol{S}_{\boldsymbol{N}}^{+} \mathbf{X}}\left(\pi_{\boldsymbol{N} \mid \mathbf{X}}^{-1} \mathbb{R} \mathscr{H}_{a m_{\mathscr{D}_{\mathbf{X}}}}\left(\mathscr{H}, \mathcal{O}_{\mathbf{X}}\right)\right)=0
$$

et

$$
\mathbb{R} \mathscr{H}_{a m_{\mathscr{D}_{\mathbf{X}}}}\left(\mathscr{H}, \mathcal{O}_{\mathbf{X}}\right) \mid \mathbb{N}=\mathbb{R} \Gamma_{\mathbf{N}} \mathbb{R} \mathscr{H}_{a m_{\mathscr{T}_{\mathbf{X}}}}\left(\mathscr{M}, \mathcal{O}_{\mathbf{X}}\right)\left[\operatorname{codim}_{\mathbf{X}} \mathbb{N}\right] \otimes \omega_{\mathbb{N} \mid \mathbf{X}} .
$$

Démonstration. Le corollaire se déduit du théorème 3.1 .4 et du théorème 4.1.2.

q.e.d.

Soit maintenant $\Omega$ un ouvert de $\mathbb{X}$ dont la frontière $\mathbb{N}=\partial \Omega$ est une hypersurface analytique réelle. On désigne par $\mathbb{F}$ le complement de $\Omega$ dans $\mathbb{X}$. On a alors le

Corollaire 4.1.4. Dans la situation précédente, si $\mathbb{N}$ est non caractéristique par rapport à $\mathscr{M}$, on a alors

$$
\mathbb{R} \Gamma_{\boldsymbol{F}} \mathbb{R} \mathscr{H}_{a m} \mathscr{D}_{\mathbf{X}}\left(\mathscr{H}, \mathcal{O}_{\mathbf{X}}\right)=0 \quad \text { sur } \mathbb{N} .
$$

Remarquons que ce résultat a été établi par $M$. Kashiwara [17] et $\mathbb{M}$. Kashiwara-T. Kawai [21] sans l'hypothèse " $\mathbb{N}$ est analytique".

Soit $\mathbb{N}$ l'hypersurface analytique réelle de $\mathbb{X}$ constituée par le bord de $\Omega$. On désignera par $\mathbb{N}_{+}$le fibré conormal extérieur de $\mathbb{N}$ en sphères et par l'inclusion naturelle de $\Omega$ dans $\mathbb{X}$. On pose $\pi=\left(\pi_{N \mid \mathbb{X}}\right) \mid \mathbb{N}_{+}$. P. Pallu de la Barrière [34] a démontré le résultat suivant dans le cas d'une seul opérateur. Nous réussissons à obtenir le résultat suivant en utilisant le théorème 3.1.4.

Théorème 4.1.5. Si le système .ll satisfait les conditions suivantes:

$$
\mathscr{E}^{\mathscr{E}} \ell_{\mathscr{D}_{\mathbf{X}}}^{k}\left(\mathscr{l}, \mathcal{O}_{\mathbf{X}}\right)=0 \quad \text { quel que soit } k>0,
$$

on a les isomorphismes suivants:

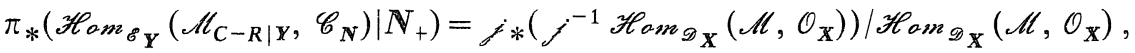

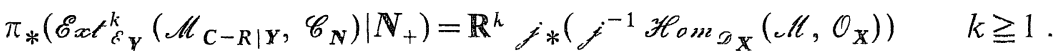

Autrement dit, la structure d'obstructions du prolongement des solutions holomorphes $d u$ système $\mathscr{l l}$ est isomorphe au faisceau des solutions microfonctions du système $\mathscr{U}_{C-R \mid \boldsymbol{X}}$ sur $\mathbb{N}_{+}$. 
Démonstration. On déduit du théorème 3.1 .4 le résultat immédiatement. c.q.f.d.

Théorème 4.1.6. Soit $\mathscr{M}$ un $\mathscr{D}_{\mathbf{X}}$-module cohérent. Soit $p_{+}$appartenant à $\mathbf{N}_{+}$. On note $p=\pi\left(p_{+}\right)$l'image de $p_{+}$dans $\mathbf{N}$. Si la forme de Levi généralisée du système $\mathscr{M}_{C-R \mid \boldsymbol{Y}}$ a au moins une valeur propre strictement négative au point $p_{+}$, on a alors

$$
\mathscr{J}_{*}\left(\mathscr{J}^{-1} \mathscr{H}_{a m_{\mathscr{D}_{\mathbf{X}}}}\left(\mathscr{H}, \mathcal{O}_{\mathbf{X}}\right)\right) / \mathscr{H}_{a m_{\mathscr{O}_{X}}}\left(\mathscr{H}, \mathcal{O}_{X}\right)_{p}=0
$$

Autrement dit, tout germe de solutions holomorphes du système $\mathscr{M}$ au voisinage de $p$ sur $\Omega$ se prolonge en solution holomorphe à travers la frontière $\mathbb{N}$ dans un voisinage de $p$.

Démonstration. On désignera encore par $\pi$ la projection $\pi: \Omega \sqcup N_{-} \rightarrow X$ et par $\dot{f}$ l'inclusion $\not{f}: \Omega \rightarrow \Omega \sqcup \mathbb{N}_{-}$. On a alors la suite exacte des faisceaux $\operatorname{sur} \mathbf{N}_{-}$:

$$
\begin{aligned}
0 & \longrightarrow \pi^{-1} \mathscr{H}_{a m_{\mathscr{D}_{\mathbf{X}}}}\left(\mathscr{M}, \mathcal{O}_{\mathbf{X}}\right) \longrightarrow \mathcal{j}_{*}\left(\dot{J}^{-1} \pi^{-1} \mathscr{H}_{a m_{\mathscr{D}_{\mathbf{X}}}}\left(\mathscr{M}, \mathcal{O}_{\mathbf{X}}\right)\right)- \\
& \longrightarrow \mathscr{H}^{1}\left(\mathbf{R} \Gamma_{N_{-}}\left(\pi^{-1} \mathbf{R} \mathscr{H}_{a m_{\mathscr{D}_{\mathbf{X}}}}\left(\mathscr{M}, \mathcal{O}_{\mathbf{X}}\right)\right)\right) \longrightarrow \pi^{-1} \mathscr{E}_{x_{\mathscr{D}_{\mathbf{X}}}^{1}}^{1}\left(\mathscr{M}, \mathcal{O}_{\mathbf{X}}\right) \longrightarrow \cdots
\end{aligned}
$$

Le théorème 2.2.1 entraîne que

$$
\mathscr{H}^{1}\left(\mathbf{R} \Gamma_{N_{-}}\left(\pi^{-1} \mathbf{R} \mathscr{H}_{a m_{\mathscr{D}_{\mathbf{X}}}}\left(\mathscr{M}, \mathcal{O}_{\mathbf{X}}\right)\right)\right)_{p_{+}}^{a}=\mathscr{H}_{a m_{\mathscr{E}_{\mathbf{Y}}}}\left(\mathscr{M}_{\mathrm{C}-\mathrm{R} \mid \mathbf{Y}}, \mathscr{C}_{\mathbf{N}}\right)_{p_{+}}=0
$$

On en déduit le résultat immédiatement.

Nous allons étudier le problème du prolongement au bord qui est feuilleté par des variétés complexes.

Soit $p$ (resp. $p_{+}$) appartenant à $\mathbb{N}$ (resp. $\boldsymbol{N}_{+}$). Soit $L_{p}$ un germe d'une sous-variété complexe passant par $p$ dans $\mathbb{N}$. On désigne par $L_{p^{+}}$l'ensemble $\pi^{-1}\left(L_{p}\right)$. On désigne par $\mathscr{D}_{C-R \mid \boldsymbol{Y}}$ le système des équations de Cauchy-Riemann tangentielles sur $\boldsymbol{N}$, en désignant $\boldsymbol{Y}$ un complexifié de $\boldsymbol{N}$.

Théorème 4.1.7. Supposons que les supports des solutions microfonctions du système $\mathscr{D}_{C-R \mid Y}$ se propagent le long de la sous-variété $L_{p^{+}}$. Supposons que le système $\mathscr{M}$ satisfasse la condition suivante:

$p_{+}$appartient à l'adhérence de l'ensemble $L_{p^{+}}-S S(\mathscr{M}) \cap L_{p^{+}}$. On a alors

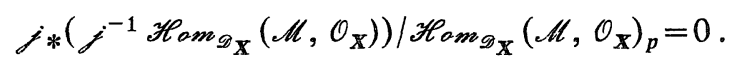

Démonstration. Par l'hypothése il y existe un ouvert $U$ de $L_{p}$ tel que 


$$
\mathscr{H}_{a m_{\mathscr{B}_{Y}}}\left(\mathscr{M}_{C-R \mid Y}, \mathscr{C}_{N}\right)_{\pi^{-1}(U)}=0 \text { et } p_{+} \in l^{\prime} \text { adhérence de } \pi^{-1}(U) .
$$

Il suffit de vérifier que les singularités des solutions du système $\mathscr{M}_{C-R \mid Y}$ se propagent le long de la sous-variété $L_{p^{+}}$. Pour cela, considérons une résolution projective locale de $\mathscr{H}$ :

$$
0 \longleftarrow \mathscr{M} \longleftarrow \mathscr{D}_{X}^{r_{0}} \longleftarrow \mathscr{D}_{X}^{r_{1}} \longleftarrow \cdots \longleftarrow \mathscr{D}_{X}^{r_{q+1}} \longleftarrow 0 .
$$

On a alors la suite exacte des systèmes suivante de façon naturelle:

$$
0 \longleftarrow \mathscr{M}_{C-R \mid Y} \longleftarrow \mathscr{D}_{C-R \mid Y}^{r_{0}} \longleftarrow \mathscr{D}_{C-R \mid Y}^{r_{1}} \longleftarrow \cdots-\mathscr{D}_{C-R \mid Y}^{r_{q+1}} \longleftarrow 0 .
$$

On en déduit le résultat facilement.

c. q. f.d.

Y. Tsuno [41] a déjà obtenu le résultat suivant dans le cas d'une seul opérateur en supposant que l'hypersurface soit Levi plat.

Théorème 4.1.8. Supposons que le système des équations de CauchyRiemann tangentiells sur le bord soit isomorphe au mélange du système de Lewy-Mizohata de la signature $(\operatorname{dim} \mathbb{X}-d-1,0)$ et $d u$ système de CauchyRiemann partiel. On désigne par $L_{p}$ un germe de la feuille analytique complexe passant par $p$, de dimension $d$. Si $p_{+}$appartient à l'adhérence de l'ensemble $L_{p^{+}}-S S(\mathscr{M}) \cap L_{p^{+}}$, on a alors

$$
\mathscr{J}_{*}\left(\dot{j}^{-1} \mathscr{H}_{a m_{D_{X}}}\left(\mathscr{H}, \mathcal{O}_{\mathbf{X}}\right)\right) / \mathscr{H}_{a_{m_{D_{X}}}}\left(\mathscr{M}, \mathcal{O}_{\mathbf{X}}\right)_{p}=0 .
$$

Démonstration. Le théorème se déduit du théorème 4.1.7.

B) Nous allons étudier le problème de la non hypo-ellipticité analytique du système tangentiel.

Soit $\mathbb{N}_{+}$le fibré conormal extérieur au bord d'un ouvert $\Omega$, ce bord $\mathbb{N}$ étant supposé analytique réel. Soit $p_{+}$la conormal extérieur à $\mathbb{N}$ en $p \in \mathbb{N}$. On note $\mathbb{Y}$ un complexifié de $\mathbb{N}$ et $j$ l'inclusion de $\Omega$ dans $\mathbb{X}$. Nous obtenons d'abord le

Théorème 4.2.1. Etant donné un $\mathscr{D}_{\mathbf{X}}$-module cohérent $\mathscr{M}$, si $\Omega$ est un domaine d'holomorphie des solutions holomorphes du système $\mathscr{M}$ en p, i.e.

$$
j_{*}\left(j^{-1} \mathscr{H}_{a m_{\mathscr{D}_{\mathbf{X}}}}\left(\mathscr{M}, \mathcal{O}_{\mathbf{X}}\right)\right) / \mathscr{H}_{a m_{\mathscr{D}_{\mathbf{X}}}}\left(\mathscr{M}, \mathcal{O}_{\mathbf{X}}\right)_{p} \neq 0,
$$

on a les propriétés suivantes:

(1) $\mathbb{N}$ est caractéristique par rapport à $\mathscr{M}$ en $p \in \mathbb{N}$.

(2) $\mathbb{N}_{+}$est pseudoconvexe par rapport à $\mathscr{M}$ en $p_{+}$au sens de S-K-K. 
Plus précisément, la forme de Levi du système $\mathscr{M}_{C-K \mid \boldsymbol{Y}} n^{\prime} a$ pas de valeurs propres strictement négatives en $p_{+} \in \mathbf{N}_{+}$.

Démonstration. Le première partie de l'énoncé resulte du corollaire 4.1.3. Quant à la deuxième partie, on déduit du théorème 4.1.6 le résultat immédiatement.

c. q.f.d.

Soit maintenant $\mathscr{M}$ un système d'équations aux dérivées partielles à coefficients constants sur $C^{m}$. Rappelons d'abord le résultat suivant dû à $H$. Komatsu [28]:

$$
\mathscr{E}_{x} t_{\mathscr{C}^{m}}^{k}\left(\mathscr{M}, \mathcal{O}_{C^{m}}\right)=0 \quad \text { quel que soit } k>0 .
$$

Choisissons un covecteur caractéristique $\zeta$ du système $\mathscr{M}$. On pose:

$$
\mathbb{N}_{\zeta}=\left\{z \in C^{m} \mid \operatorname{Re}\langle z, \zeta\rangle=0\right\} \text { et } \Omega_{\zeta}=\left\{z \in C^{m} \mid \operatorname{Re}\langle z, \zeta\rangle>0\right\} .
$$

On note $j$ l'inclusion naturelle de $\Omega_{\zeta}$ dans $C^{m}$. On a alors la

Proposition 4.2.2. (C. O. Kiselman [26]). Dans la situation précédent, supposons que $\mathscr{M}$ soit de la forme suivante:

$$
\mathscr{M}=\mathscr{D}_{C^{m}} u=\mathscr{D}_{C^{m}} / \mathscr{J} \quad \text { où } \quad \mathscr{J}=\left\{P \in \mathscr{D}_{C^{m}} \mid P u=0\right\} .
$$

Alors le système $\mathscr{M}_{C-R \mid \boldsymbol{Y}}$ est non-hypo-elliptique analytique.

Démonstration. En utilisant une mèthode dûeà C. O. Kiselman on peut construire concrètement une fonction holomorphe sur $\Omega_{\zeta}$ de sorte qu'elle

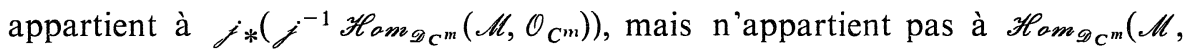
$\left.\mathcal{O}_{C^{m}}\right)$. Ceci entraîne le résultat.

q.e.d.

Conjecture 4.2.3. (Non hypo-ellipticité analytique microlocal). Etant donné un système $\mathscr{M}$ d'équations aux dérivées partielles à coefficients constants sur $C^{m}$, supposons que un point $q \in T_{R^{m}}^{*} C^{m}-T_{R^{m}}^{*} R^{m}$ appartient à $S S(\mathscr{M})$. Alors il y existe une microfonction (non nulle) verifiant le système.

Soient $\Omega_{1} \subseteq \Omega_{2}$ deux ouverts de $\boldsymbol{X}$. On suppose que les bords $\mathbb{N}_{1}$ et $\mathbb{N}_{2}$ soient les hypersurfaces analytiques réelles. On suppose aussi que le bord $\mathbb{N}_{2}$ soit tangent au bord $\mathbb{N}_{1}$ en $p \in \mathbb{N}_{1} \Gamma_{1} \mathbb{N}_{2}$. On désigne par $\mathbb{N}_{1+}\left(\operatorname{resp} . \mathbb{N}_{2+}\right)$ le fibré conormal extérieur à $\mathbb{N}_{1}$ (resp. $\mathbb{N}_{2}$ ) et par $p_{+}$la conormale extérieur commune en $p$. On note $\boldsymbol{Y}_{1}$ (resp. $\left.\boldsymbol{Y}_{2}\right)$ un complexifié de $\boldsymbol{N}_{1}\left(\operatorname{resp} . \boldsymbol{N}_{2}\right)$.

On est alors en mesure de obtenir le résultat suivant:

Théorème 4.2.4. Soit $\mathscr{M}$ le $\mathscr{D}_{\mathbf{X}}$-module cohérent vérifiant la condition suivante: 


$$
\mathscr{E}_{x \rightarrow \mathscr{T}_{\mathbf{X}}}^{1}\left(\mathscr{M}, \mathcal{O}_{\mathbf{X}}\right)=0
$$

On a alors

$$
\mathscr{H}_{a m_{\mathscr{O}_{Y_{2}}}}\left(\mathscr{M}_{C-R \mid \mathbf{Y}_{2}}, \mathscr{C}_{\mathrm{N}_{2}}\right)_{p_{+}} \subseteq \mathscr{H}_{a m_{\mathscr{O}_{Y_{1}}}}\left(\mathscr{M}_{C-R \mid Y_{1}}, \mathscr{C}_{N_{1}}\right)_{p_{+}} .
$$

En particulier, si le système $\mathscr{M}_{C-R \mid \mathbf{Y}_{2}}$ est non-hypo-elliptique en $p_{+}$, le système $\mathscr{M}_{C-R \mid Y_{1}}$ est aussi non-hypo-elliptique en $p_{+}$.

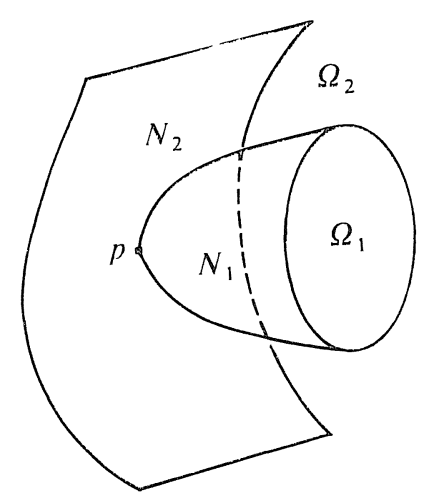

Démonstration. On désigne par $j_{q}(q=1,2)$ l'inclusion $j_{q}: \Omega_{q} \rightarrow \mathbb{X}$. On a alors les isomorphismes suivants:

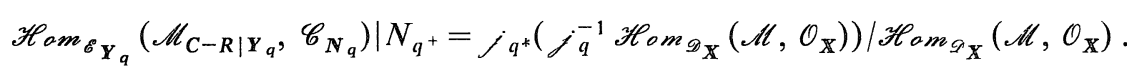

On en déduit le résultat tout de suite.

c. q.f.d.

Exemple 4.2.5. Soit $\mathscr{l}$ un système d'équations aux dérivées partielles à coefficients constants sur $C^{m}$, satisfaisant la condition de la proposition 4.2.2. Choisissons un vecteur caractéristique $\zeta$ du système $\mathscr{H}$ et le demi-espace $\Omega_{2}$ $=\Omega_{\zeta}=\left\{z \in C^{m} \mid \operatorname{Re}\langle z, \zeta\rangle>0\right\}$ de la frontière $\mathbb{N}_{2}=\mathbb{N}_{\zeta}$. Soit $\Omega_{1}$ un ouvert de $\Omega_{2}$, ce bord $\mathbb{N}_{1}$ étant supposé analytique réel. Supposons que $\mathbb{N}_{2}$ soit tangent à $\mathbb{N}_{1}$ en $p$. Alors le système $\mathscr{M}_{C-R \mid \boldsymbol{Y}_{1}}$ n'est pas hypo-elliptique analytique en $p_{+}$.

Théorème 4.2.6. Plaçons-nous dans la situation du théorème 4.2.4.

(1) Supposons que tout germe de soutions holomorphes sur $\Omega_{1}$ se prolonge $\grave{a} \Omega_{2}$ au voisinage de $p$, i.e.

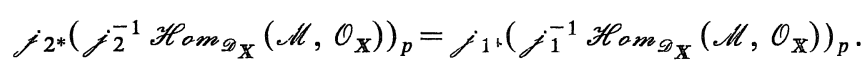

On a alors l'isomorphisme naturel suivant en $p_{+}$: 


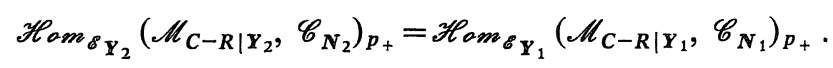

(2) Supposons de plus que

$$
\begin{aligned}
& \mathscr{E}_{x^{t}}^{k}\left(\mathscr{M}, \mathcal{O}_{\mathbf{X}}\right)=0 \quad \text { quel que soit } k \geqq 1,
\end{aligned}
$$

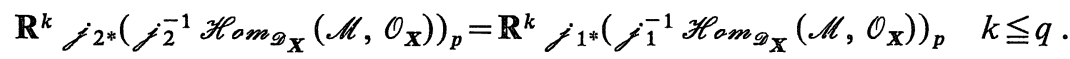

Alors

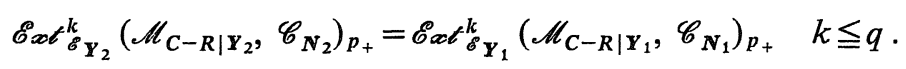

Démonstration. On déduit de la démonstration du théorème 4.2 .4 et du théorème 4.1.5 le résultat immédiatement.

c. q.f.d.

On peut rapprocher ce résultat d'un résultat profond de S-K-K (Chap. 3, Lemme 2.3.7). En utilisant le théorème 4.2.6, on peut généraliser ce résultat de $\mathrm{S}-\mathrm{K}-\mathrm{K}$ de façon naturelle. Si le système $\mathscr{M}$ est un système régulier au sens de S-K-K, on peut traiter concretement le système $\mathscr{M}_{C-R \mid Y}$ sans l'hypothése "la forme de Levi généralisée de $\mathscr{M}_{C-R \mid Y}$ garde un rang constant". Nous voulons terminer en donnant quelques exemples. On pose:

$$
\begin{aligned}
& X=C^{2}=\left\{\left(z_{1}, z_{2}\right) \mid z_{1}=x_{1}+i y_{1}, z_{2}=x_{2}+i y_{2} \in C\right\}, \\
& \Omega=\left\{\left(z_{1}, z_{2}\right) \mid x_{2}<0\right\}, \mathbb{N}=\partial \Omega, \mathscr{M}=\mathscr{D}_{\mathbf{x}} / \mathscr{D}_{\mathbf{x}} \frac{\partial}{\partial z_{1}} .
\end{aligned}
$$

Il est evident que le système $\mathscr{M}_{C-R \mid Y}$ coïncide avec le système de de Rham partiel:

$$
\frac{\partial}{\partial x_{1}} u=\frac{\partial}{\partial y_{1}} u=0 \text {. }
$$

Exemple 4.2.7. Plaçons-nous dans la situation précédente.

(1) On pose: $\Omega_{1}=\left\{\left(z_{1}, z_{2}\right) \mid x_{2}+x_{1}^{2 l}+y_{1}^{2 m}<0\right\}$, où $l, m \geqq 1 . \quad \mathbf{N}_{1}=\partial \Omega_{1}$. Il est facile de voir que

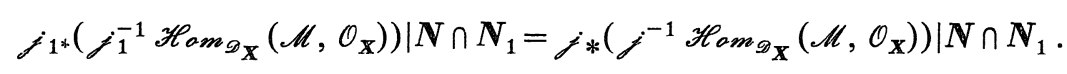

On obtient donc

$$
\begin{aligned}
& \mathscr{H}_{a m_{\mathscr{B}_{Y_{1}}}}\left(\mathscr{M}_{C-R \mid \mathbf{Y}_{1}}, \mathscr{C}_{\boldsymbol{N}_{1}}\right)\left|\mathbb{N}_{+} \cap \mathbb{N}_{1^{+}}=\mathscr{H}_{a m_{\mathscr{E}_{Y}}}\left(\mathscr{M}_{C-R \mid Y}, \mathscr{C}_{N}\right)\right| \mathbb{N}_{+} \cap \mathbf{N}_{1+} \\
& =\mathscr{C}_{\boldsymbol{N}_{+} \cap \boldsymbol{N}_{1_{+}}} \cong \mathscr{C}_{\boldsymbol{R}_{+}} \text {. }
\end{aligned}
$$

(2) On pose: $\Omega_{2}=\left\{\left(z_{1}, z_{2}\right) \mid x_{2}-x_{1}^{2 l}-y_{1}^{2 m}<0\right\}$ où $l, m \geqq 1$, et $\mathbf{N}_{2}=\partial \Omega_{2}$. D'après le théorème 4.2 .4 , on a

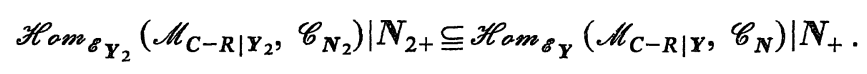

Il est evident que 


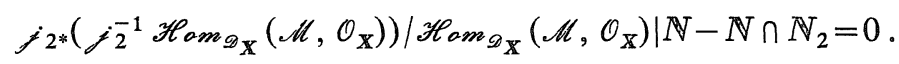

D'autre part, les singularités des solutions du système $\mathscr{M}_{C-R \mid Y}$ se propagent au long de les variétés caractéristiques. On obtient donc

$$
\mathscr{H}_{\text {am }_{{ }_{B}} \mathbf{Y}_{2}}\left(\mathscr{M}_{\mathrm{C}-\mathrm{R} \mid \mathbf{Y}_{2}}, \mathscr{C}_{\mathrm{N}_{2}}\right) \mid \mathbb{N}_{2+}=0 .
$$

(3) On pose: $\Omega_{3}=\left\{\left(z_{1}, z_{2}\right) \mid x_{2}+x_{1}^{2 l}+y_{1}^{2 m-1}<0\right\}$ où $l, m \geqq 1$, et $\mathbb{N}_{3}=\partial \Omega_{3}$. On désigne par $\hbar$ l'inclusion de $\Omega \cup \Omega_{3}$ dans $\mathbb{X}$. Il est facile de voir que

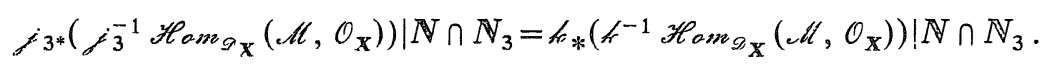

On a alors

$$
\mathscr{H}_{a m_{\mathscr{E}_{Y_{3}}}}\left(\mathscr{M}_{C-R \mid \mathbf{Y}_{3}}, \mathscr{C}_{N_{3}}\right)\left|\mathbb{N}_{+} \cap \mathbb{N}_{3+} \subseteq \mathscr{H}_{a m_{\mathscr{E}_{Y}}}\left(\mathscr{M}_{C-R \mid Y}, \mathscr{C}_{N}\right)\right| \mathbb{N}_{+} .
$$

On en déduit que

$$
\mathscr{H}_{a m_{\mathscr{E}_{Y_{3}}}}\left(\mathscr{M}_{C-R \mid \boldsymbol{Y}_{3}}, \mathscr{C}_{N_{3}}\right) \mid \mathbb{N}_{3+}=0
$$

\section{Bibliographie}

[1] Andreotti, A. et Fredricks, G. A. Embeddability of real analytic Cauchy-Riemann manifold, Ann. Scuola Norm. Sup. Pisa, 6 (1979), 285-304.

[2] Andreotti, A. et Grauert, $H$. Théorèmes de finitude pour la cohomologie des espaces complexes, Bull. Soc. Math. France, 90 (1962), 193-259.

[3] Andreotti, A. et Hill, C. D. Complex characteristic coordinates and tangential Cauchy-Reimann equations, Ann. Scuola Norm. Sup. Pisa, 26 (1972), 299-324.

[4] -., E. E. Levi convexity and the E. Lewy problem I, II, Ann. Scuola Norm. Sup. Pisa, 26 (1972), 323-363, 747-806.

[5] Andreotti, A. et Nacinovich, M. Analytic convexity, Ann. Scuola Norm. Sup. Pisa, 7 (1980), 287-372.

[6] Bony, J. M. et Schapira, P. Existence et prolongement des solutions holomorphes des équations aux dérivées partielles, Inventiones Math., 17 (1972), 95-105.

[7] Freeman, M., Local complex foliation of real submanifold, Math Ann., 209 (1974), $1-30$.

[8] Greenfield, S. J., Cauchy-Riemann equations in several variables, Ann. Scuola Norm. Sup. Pisa, 22 (1968), 275-314.

[9] Hartshorne, R., Residues and duality, Springer Lecture Notes in Math., 20 (1966).

[10] Harvey, F. R., Hyperfunctions and linear partial differential equations, Proc. Nat. Acad. Sci. U. S. A., 55 (1966), 1042-1046.

[11] -. The theory of hyperfunctions on totally real subsets of a complex manifold with applications to extension problems, Amer. J. Math., 91 (1969), 853-873.

[12] Kaneko, A., On continuation of regular solutions of partial differential equations to compact convex sets II, J. Fac. Sci. Univ. Tokyo, Sec IA, 18 (1972), 415-433.

[13] -., On continuation of regular solutions of partial differential equations with constant coefficients, J. Math. Soc. Japan, 26 (1974), 92-123. 
[14] Kaneko, A., On linear exceptional sets of solutions of linear partial differential equations with constant coefficients, Publ. RIMS, Kyoto Univ., 且1 (1976), 441-460.

[15] Kashiwara, M., Algebraic study of systems of partial differential equations, Master's thesis, Univ. of Tokyo, 1971 (Japanese).

[16] -., On the maximally overdetermind systems of linear differential equations I, Publ. RIMS, Kyoto Univ., 10 (1975), 563-579.

[17] -. Systèmes d'équations micro-différentielles, Notes de Teresa Monteiro Fernandes, Univ. Paris-Nord, (1978).

[18] Kashiwara, M. et Kawai, T. On the boundary value problem for elliptic systems of linear differential operators I, II, Proc. Japan Acad., 48 (1972), 712-715, ibid 49 (1973), 164-168.

[19] -., Theory of elliptic boundary value problems and its applications, SûrikaisekiKenkyûsho Kôkyûroku, 238 Kyoto Univ. (1975), 1-59, (Japanese).

[20] -., Some appiications of boundary value problems for elliptic systems of linear differential equations, Annales of Math. Studies, Princeton Univ. Press, 93 (1979), 39-61.

[21] -., On holonomic systems of microdifferential equations III, RIMS Preprint, Kyoto Univ., 293 (1979).

[22] Kashiwara, M. et Schapira, P. Micro-hyperbolic systems, Acta. Math., 142 (1979), $1-55$.

[23] Kawai, T., Finite dimensionality of cohomology groups attached to systèms of linear differential equations, J. Math. Kyoto Univ., 13 (1973), 73-95.

[24] -. Vanishing of cohomology groups on completely k-convex sets, Publ. RIMS, Kyoto Univ., 11 (1976), 775-784.

[25] -. Extension of solutions of systems of linear differential equations, Publ. RIMS, Kyoto Univ., 12 (1976), 215-227.

[26] Kiselman, C. O., Prolongement des solutions d'une équation aux dérivées partielles à coefficients constants, Bull. Soc. Math. France 97 (1969), 329-356.

[27] Kohn, J. J., Subellipticity of the $\bar{\partial}$-Neumann problem on pseudo-convex domains (sufficient conditions), Acta. Math., 142 (1979), 79-122.

[28] Komatsu, H., Resolutions by hyperfunctions of sheaves of solutions of differential equations with constant coefficients, Math. Annalen, 176 (1968), 77-86.

[29] Komatsu, H. et Kawai, T. Boundary values of hyperfunction solutions of linear partial differential equations, Publ. RIMS, Kyoto Univ., 7 (1971/72), 95-104.

[30] Lewy, H., An example of a smooth linear partial differential equation without solution, Annales of Math., 66 (1957), 155-158.

[31] Martineau, A., Le "edge of the wedge theorem" en théorie des hyperfonctions de Sato, Proc. Intern. Conf. on Functional Analysis and Related Topics, Tokyo 1969, Univ. Tokyo Press (1970), 95-106.

[32] Naruki, I., An analytic study of a pseudo-complex structure, Proc. Intern. Conf. on Functional Analysis and Related Topics, Tokyo 1969, 72-82.

-, Holomorphic extension problem for standard real submanifolds of second kind, Publ. RIMS, Kyoto Univ., 6 (1970), 113-187.

[33] -. Localization principle for differential complexes and its applications, Publ. RIMS, Kyoto Univ., 8 (1972), 43-110.

[34] Pallu de la Barrière, P., Existence et prolongement des solutions holomorphes des équations aux dérivées partielles, J. Math. Pures et Appl., 55 (1976), 21-46.

[35] Persson, J., Local analytic continuation of holomorphic solutions of partial differential equations, Ann. Mat. Pura Appl., 112 (1977), 193-204. 
[36] Polking, J. C. et Wells, R. O., Jr., Boundary values of Dolbeault cohomology classes and a generalised Bochner-Hartogs theorem, Abhand Math. Sem. Univ. Hamburg, 47 (1978), 1-24.

[37] Sato, M., Kawai, T. el Kashiwara, M. (S-K-K), Microfunctions and pseudo-differential equations, Springer Lecture Notes in Math., 287 (1973), 265-529.

[38] Schapira, P., Conditions de positivité dans une variété symplectique complexe. Applications à l'étude des microfonctions, Ann. Scient. Ec. Norm. Sup., 14 (1981), 121-139.

[39] Tomassini, G., Tracce delle funzioni olomorfe sulle sotto-varieta analitiche reali d'una varietà complessa, Ann. Scuola Norm. Sup. Pisa, 20 (1966), 31-43.

[40] Tsuno, Y., On the continuations of holomorphic solutions of characteristic differential equations, J. Math. Soc. Japan, 26 (1974), 523-548.

[41] -. On the prolongation of local holomorphic solutions of partial differential equations II, prolongations across the pluri-harmonic hypersurface. J. Math. Soc. Japan, 28 (1976), 304-306.

[42] -. Holomorphic continuation of solutions of partial differential equations across the multiple characteristic surface, J. Math. Soc. Japan, 32 (1980), 285-299.

[43] - Localization of differential operators and holomorphic continuation of the solutions, Hiroshima Muth. J., 10 (1980), 539-551.

[44] Wells, R. O., Jr., Hyperfunction solutions of the zero-restmass field equations, Commun. Math. Phys., 78 (1981), 567-600.

[45] Zerner, M., Domaine d'holomorphie des fonctions vérifiant une équation aux dérivées partielles, C. R. Acad. Sci. Paris, 272 (1971), 1646-1648.

[46] Andreotti, A., Fredricks, G. et Nacinovich, M., On the absence of Poincaré lemma in tangential Cauchy-Riemann complexes, Ann. Scuola Norm. Sup. Pisa, 8 (1981), 365-404.

[47] Henkin, G. M., Solution des équations de Cauchy-Riemann tangentielles sur des variétés de Cauchy-Riemann $q$-concaves, C. R. Acad. Sc. Paris, 292 (1981), 27-30. 
